\title{
The effect of assembly and static unbalance on reaction wheel assembly bearing harmonics
}

\author{
Cameron Hodge $^{1}$ (i) $\cdot$ Alessandro Stabile ${ }^{1} \cdot$ Guglielmo Aglietti $^{2} \cdot$ Guy Richardson $^{3}$
}

Received: 18 August 2020 / Revised: 12 November 2020 / Accepted: 4 December 2020 / Published online: 28 December 2020

(c) The Author(s) 2020

\begin{abstract}
Reaction wheel assemblies (RWA) are well-known major sources of microvibrations, whilst they have been studied thoroughly and many disturbance types can be reasonably modelled, bearing disturbances and how their amplitude evolves with the RWA rotational speed are not at the same level of confidence. Whilst studies have been carried out, many of the test rigs used do not truly show the bearing harmonic development, either due to interference from other disturbances such as structural modes or are not representative of an RWA. This study aims to design and validate a test rig which alleviates those issues by moving the resonance frequencies out of a range of interest and isolating the motor disturbances. Using this test rig, it was possible to observe many engine order development without any inference and start to investigate some of the effects some manufacturing parameters can have. The two studied and discussed in this paper were the effect of reassembly and static unbalance. Investigating the microvibration signature at different levels ranging from a top-down to individual harmonics it showed a clear significant variation between disturbance amplitudes.
\end{abstract}

Keywords Bearings $\cdot$ Localised defect $\cdot$ Microvibration $\cdot$ Reaction wheel assemblies

\section{Introduction}

Low-level mechanical disturbances also known as microvibrations have become an increasingly important aspect in the design of scientific satellites. Microvibrations usually have an amplitude in the range of $\mu \mathrm{g}$ 's and typically occur up to $1 \mathrm{kHz}[1,2]$. These disturbances are especially concerning for the design of satellites used in earth observation and space astronomy as they can cause poor pointing stability and imaging, leading to adverse effects on the satellites' instrumentation [3-5]. Fig. 1 shows a visual example of how imaging instruments can be affected by these disturbances. The left image represents a comparison between the maximum desired displacements (red circle) against the actual (blue lines). The right three photos compare images taken

Cameron Hodge

c.hodge@surrey.ac.uk

1 Surrey Space Centre, University of Surrey, Guildford GU2 7XH, England

2 The University of Auckland, Auckland Central, Auckland 1010, New Zealand

3 Surrey Satellite Technology Ltd, 20 Stephenson Road, Guildford GU2 7YE, England when significant microvibrations were present, when some post processing had been applied and when the imager was undisturbed during the capture process [6]. Even though corrective measures can be taken to smooth the effects the resultant image is still not as clear, as without prevalent microvibrations.

Mechanisms with rotating components such as reaction wheel assemblies (RWA) are typically one of the more dominant sources of microvibrations [7], whilst precautions are taken to reduce defects during manufacture, as with many mechanical systems there will always be slight imperfections due to tolerances which can produce disturbances during operation [8]. Granted other mechanisms such as dampers can be used to minimise the effects of these vibrations [9], this is not ideal as it will increase the overall cost and weight of a payload. As a result, there is continuing research being carried out to understand and reduce the noise generated at the source.

Previous studies have shown that the disturbances created within wheel assemblies mostly come from four main sources, the unbalance, structural modes of the disk-shaft assembly, motor and bearing disturbances [10,11].

From the literature is can be seen that models have been created to determine both the frequency and amplitude for 


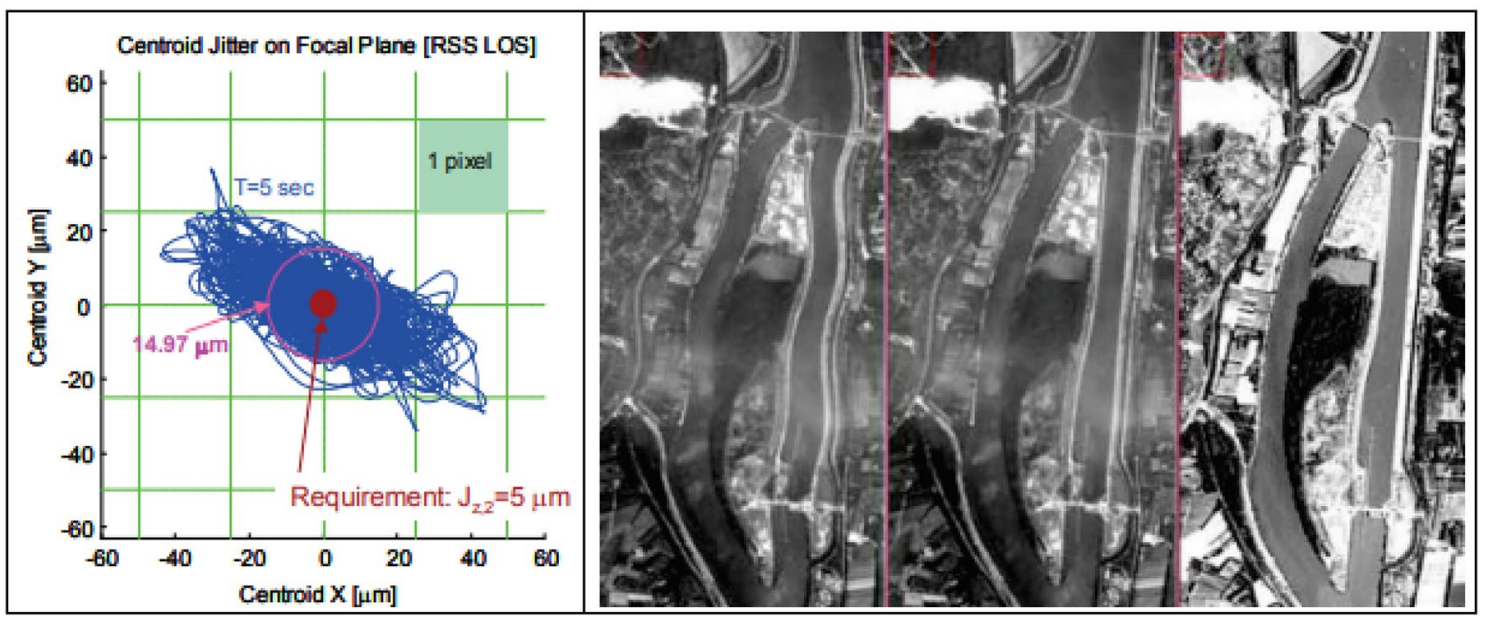

Fig. 1 Line of Sight pointing as a function of time (left) and resulting effects on the image quality (right) [6]

the unbalance and structural modes [12], however much work is needed to predict and understand what influences the amplitude of the bearing disturbances. Although certain parameters surrounding the bearing imperfections such as defect shape [13] are thought to have a direct impact on the disturbances much work is still required to improve the models to a point in which they could accurately predict the vibration amplitude in relation to the rotational speed of the wheel assembly.

Although there have been many studies specifically focused on these vibrations, many use an experimental setup which either uses a simpler setup (examples can be seen in Fig. 2) or a full reaction wheel. Whilst a simplified setup is useful in characterizing specific details of a single

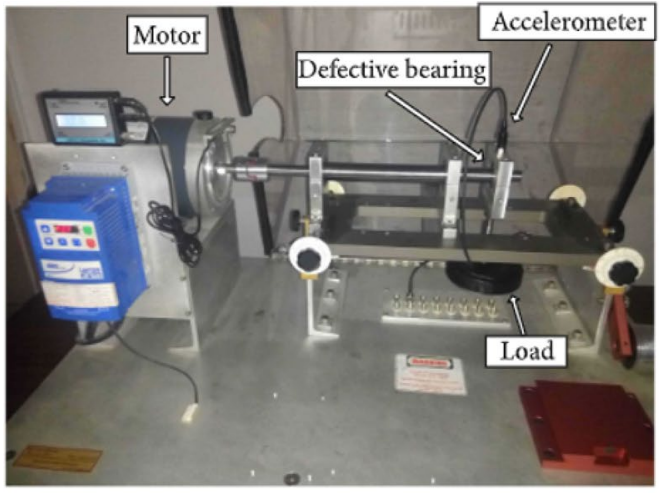

(a)

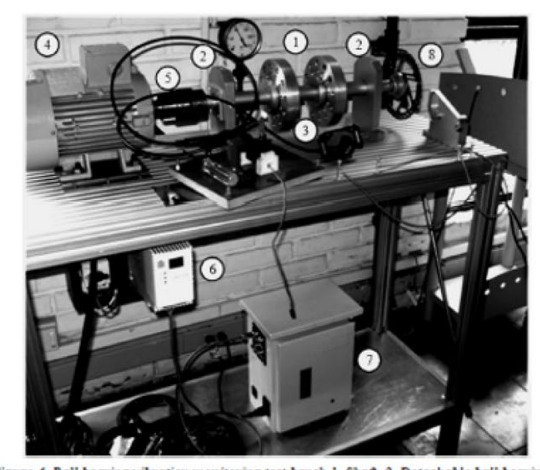

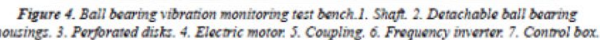

(b)

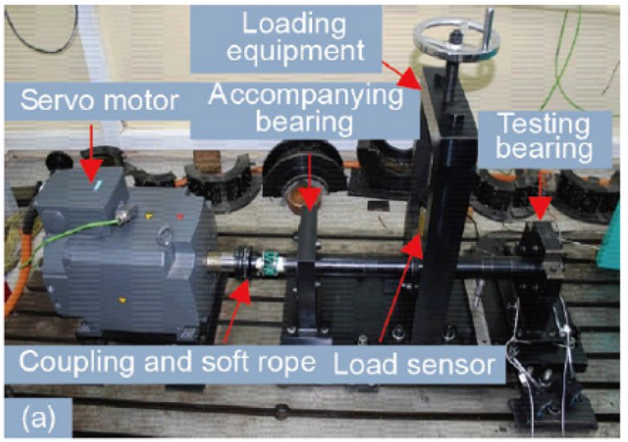

(c)

Fig. 2 Examples of a "simplified" bearing test setups a [14] b [15] c [16] 
bearing, they don't typically have many of the features seen within a reaction wheel assembly, such as the preload, multiple bearings or inertia disk. In addition many record vibrations in a limited number of degrees, for example Fig. 2a uses only one accelerometer positioned directly on the bearing housing in the vertical direction, whereas Fig. $2 b$ uses two, one for the vertical and another for the horizontal. Whilst in some cases this number of degrees of freedom might be sufficient however for a mechanism like a reaction wheel which must be made to such high levels of precision, slight discrepancies in the radial directions must be considered. Additionally, based on the position of the bearings in relation to the transducers, moments may need to be recorded. Therefore, it may be required to record the vibrations in six degrees of freedom. However, using a full reaction wheel introduces all the other known disturbances, some of which interfere with the bearings making it difficult to accurately measure and create models to predict the vibration amplitudes. This paper discusses a test rig known as the Kistler bearing test (KBT) which was designed to overcome these issues allowing observations on how bearing disturbances evolve with respect to the rotational speed without interference. As well as the methodology used to measure and analyse the microvibration response with a heavy focus on the disturbances originating from deep groove ball bearings.

\subsection{Reaction wheel disturbances}

To produce a test rig which simulates an RWA but creates an interference-free region, it is imperative to understand the different major disturbance sources. As mentioned previously the known disturbances can be classified into four groups, the structural modes, unbalance, bearing disturbances and motor disturbances.

Structural modes describe the motion of the system when excited at the resonant frequencies. In the case of RWA these can come in three forms, an axial, lateral or rocking whirl mode [17, 18], Fig. 3 shows a visual representation of what these modes could look like. RWA typically come in two configurations, symmetric and cantilever, symmetric having the centre of mass of the disk-shaft assembly in the middle of a set of bearings [9] whilst a cantilever will be located at the end. Depending on the configuration the structural modes may appear slightly different. Symmetric RWA modes will appear as distinct lateral, axial or whirl motions. However, in a cantilever configuration the lateral and rocking modes are combined. Pure axial and pure lateral modes occur are single frequencies which are determined by the geometric and material properties of the system [19]. Although whirl modes start at a singular frequency as the inertia disk accelerates the mode splits into two known as the precession and nutation whirls [18] with the precession frequency increasing and the nutation decreases. (Fig. 4).
Fig. 3 Structural modes of a reaction wheel assembly taken from [20]

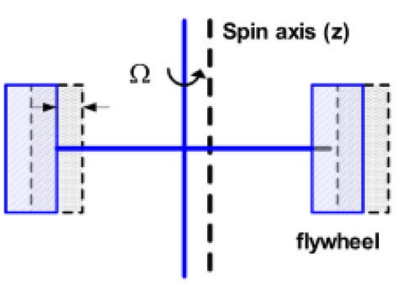

Lateral mode ( $x$ and $y$ )

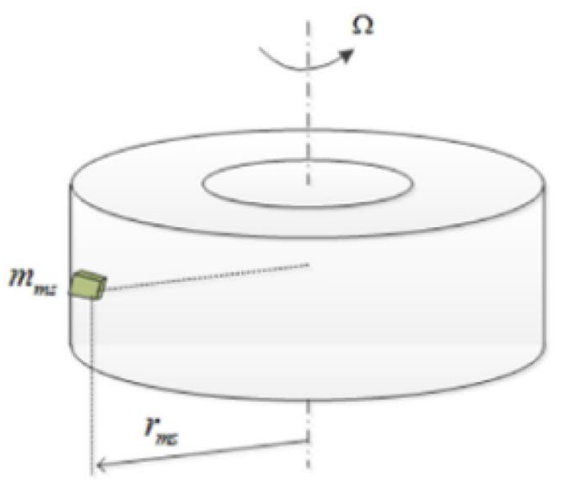

(a)

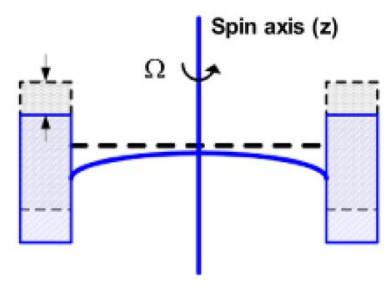

Axial mode

(z)

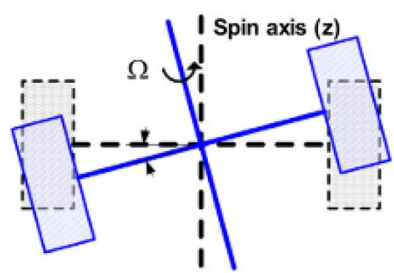

Rocking whirl mode $(\theta$ and $\Phi)$
Fig. 4 Diagram showing the effects of the static and dynamic unbalance on the axis of inertia taken from [23]

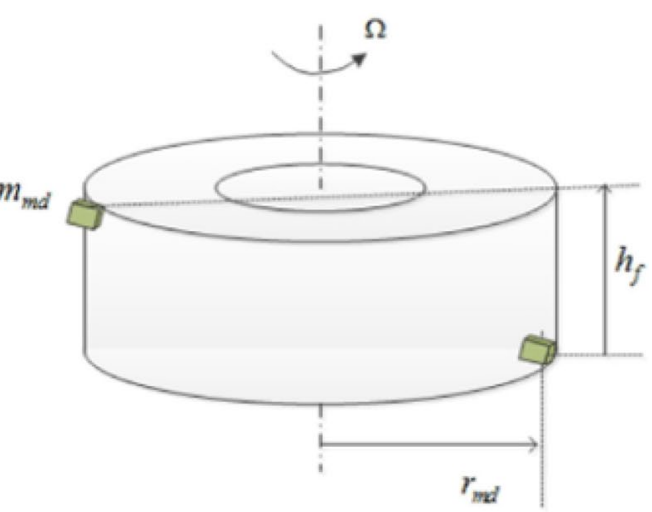

(b) 
Because of this, these modes will typically intersect the other disturbances at higher wheel speeds creating interference with higher engine order disturbances, this makes it difficult to truly understand how the bearing disturbances develop with the wheel speed.

The unbalance can be created from several potential reasons such as non-uniform mass distributions or the misalignment of rotating objects during assembly. In the case of an RWA, the largest contributor would be from the disk-shaft assembly. Either of these unbalance problems can create a shift in the axis of inertia from the axis of rotation, as a result centrifugal forces are produced and can be measured through vibrations with the same frequency as the rotational speed [21]. To reduce this disturbance, it is common practise to either remove or add additional mass to shift the axis of inertia to fit better with the axis of rotation [22]. There are two forms the unbalance can take, the static and dynamic. Pure static unbalance is where the mass distribution causes the axis of inertia to stay parallel to the axis of rotation shifting the centre of mass along one plane. Whilst in the case of pure dynamic unbalance the centre of mass remains along the axis of rotation, but the axis of inertia is rotated, creating equal and opposite forces at different axial positions. Meaning you cannot observe the dynamic through solely looking at the forces instead moments must be measured.

Whilst it is known that bearing disturbances and loads are mostly created through microscopic defects [24], aside from the defect size there are many other potential parameters that can have an influence, such as bearing geometry, material or even the system configuration [25-27]. Imperfections in bearings can be categorised into two types, localised or continuous [28]. Continuous defects tend to encompass large areas that occur during manufactures such as surface roughness or waviness. The disturbances created by continuous defects can be difficult to identify as they appear as almost "broadband" noise with no distinct frequency. However, what does separate waviness from surface roughness is the wavelength, if compared to the rolling element-raceway contact width or less then the defect is termed surface roughness if larger then waviness [29]. Localised defects however are typically isolated to small areas and consist of imperfections such as pits, spalls and cracks [30]. These disturbances come in the form of repeated impulses that occur at regular intervals. Unlike continuous disturbances, these occur at distinct frequencies proportional to the rotational speed of the mechanism, additionally as these originate from impulses subsequent frequencies can be observed at integer multiples or orders of the fundamental frequency known as harmonics. The fundamental harmonic refers to the first frequency a harmonic disturbance creates. Figure 5 shows a representation of how the harmonics typically compare to the fundamental. Depending on the origin point of the disturbance and location of the force transducers recording, sidebands

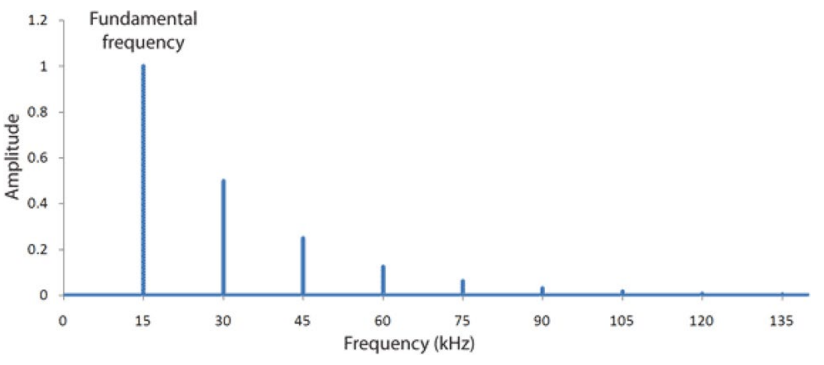

Fig. 5 Representation of how harmonics can be observed in the frequency domain

can be observed as the harmonics interfere with each other. Sidebands take the form of additional engine orders with frequencies that are the original disturbances plus or minus the interfering.

To bridge the gap between the "simplified" test setups and an RWA certain criteria must be discussed. Two essential criteria's which were discussed during the design process, these were that the system must resemble a RWA in that the preload method, bearing setup and operating speeds reflect in industry RWA. The other essential criteria were that the first natural frequency in the radial direction of the system must be higher than the fundamental harmonic disturbance that originates from the bearings. To satisfy both criteria it was chosen that the system would be based on a current symmetric configured RWA. This is as the two of the three structural modes are independent of the rotational speed.

\subsection{Defect detection}

As bearings used in aerospace applications are typically manufactured to such a high standard it can be incredibly difficult to visually observe any imperfection before assembly. Instead, an alternative approach through mathematical models has been created to determine the frequency of the harmonics based on the bearing geometry. Different models have been created for the many different styles of bearing, however, for this study deep groove ball bearings were used. Table 1 lists the models used to calculate the harmonic orders relative to the rotational speed of the inertia disk. Tables 2 and 3 then lists the geometric properties and calculated harmonic orders of the bearings used in this study. Whilst understanding the location of defect based on the harmonic order is useful, it does not help designers predict the amplitude of the disturbances or any information about a specific defect. Some analytical models have been created to achieve this however they are typically based on defects not representative of what would typically be seen in realworld scenarios. These models mostly focus on using Herztian contact theory to determine the stresses induced within a bearing $[14,31]$. 
Table 1 Summary of models to determine the harmonic order of each disturbance source [17, 29, 32]

\begin{tabular}{lll}
\hline Bearing disturbances & Abbreviation & Model \\
\hline Cage frequency relative to the outer race & FCOR & $\mathrm{H}_{\mathrm{COR}}=\frac{1}{2}\left(1-\frac{\mathrm{d}}{\mathrm{D}} \cos \alpha\right)$ \\
Cage frequency relative to the inner race & FCIR & $\mathrm{H}_{\mathrm{CIR}}=\frac{1}{2}\left(1+\frac{\mathrm{d}}{\mathrm{D}} \cos \alpha\right)$ \\
Ball Pass frequency of outer race (corresponding to outer race imperfection) & FBOR & FBIR \\
Ball Pass frequency of inner race (corresponding to inner race imperfection) & FBI & $\mathrm{H}_{\mathrm{BOR}}=\frac{\mathrm{z}}{2}\left(1-\frac{\mathrm{d}}{\mathrm{D}} \cos \alpha\right)$ \\
Rolling element spin frequency (corresponding to ball imperfection) & & $\mathrm{H}_{\mathrm{BI}}=\frac{\mathrm{z}}{2}\left(1+\frac{\mathrm{d}}{\mathrm{D}} \cos \alpha\right)$ \\
\end{tabular}

Where: $\mathrm{d}$ - Rolling element diameter, mm, D - Bearing pitch circle diameter, mm, $\alpha$ - Contact angle, degree, $\mathrm{z}-\mathrm{Number}$ of balls, $\mathrm{H}-\mathrm{Harmonic}$ Number

Table 2 SR8 Geometric Properties

\begin{tabular}{lll}
\hline Parameter & Value & Unit \\
\hline Number of balls & 10 & - \\
Ball diameter & 3.96875 & $\mathrm{~mm}$ \\
Bearing internal bore diameter & 12.7 & $\mathrm{~mm}$ \\
Bearing outside diameter & 28.575 & $\mathrm{~mm}$ \\
Pitch circle diameter & 20.6375 & $\mathrm{~mm}$ \\
Contact angle (loaded) & 12 & degree \\
Radial internal clearance & 0.02 & $\mathrm{~mm}$ \\
\hline
\end{tabular}

Values were taken directly from the manufacturer's datasheet

Table 3 Calculated harmonic orders based on the properties in Table 2

\begin{tabular}{ll}
\hline Disturbance & Harmonic order \\
\hline FCOR & 0.41 \\
FCIR & 0.59 \\
FBOR & 4.06 \\
FBIR & 5.93 \\
FBI & 2.51 \\
\hline
\end{tabular}

\section{Methodology and test rig design}

The tests described in this paper look at two different parameters that can be controlled during the manufacturing process. The static unbalance and whether system reassemble affects the harmonic disturbances created by the bearings. For the tests discussed in this study three pairs of bearings were used and named $\mathrm{AB}, \mathrm{CD}$ and EF. Prior to this study bearings, $\mathrm{AB}$ and $\mathrm{CD}$ had been subjected to environmental testing which involved a series of shocks and vibrations to simulate an extreme launch environment. Whereas bearings EF were assembled purely for this study and only used in the testing of the effects of reassembly. As discussed in the introduction to balance the rotor of this assembly mass can either be added or removed to reposition the axis of inertia. As removing material is a permanent solution and the tests needed to be repeatable, adding mass through the use of $\mathrm{M} 2 \times 3 \mathrm{~mm}$ grub screws was chosen. These could be added in 12 different locations spread equally along the circumference of the rotor.

\subsection{Kistler bearing test design}

To observe the disturbances produced by the bearings, a test setup coined the Kistler bearing test or KBT was designed taking inspiration from the SSTL SSW-110 RWA. The purpose of this test rig was to replicate an existing RWA but without interference from the motor disturbances or structural modes. By replacing the standard large inertia disk with something that is stiff and light weight but with enough mass to produce some inertia, raises the resonance frequencies above that of the range of interest. For this study, the frequency range of interest would need to include all of the harmonics shown in Table 3 for a given speed range, which in this case would need to cover the operational speed of the SSW-110 RWA. 0 to 6000 RPM was chosen therefore the frequency range would need to cover at least up to $593 \mathrm{~Hz}$ in the radial direction. Figure 6 shows a section view of the KBT displaying the bearing setup. Whilst it is impossible to completely remove the structural modes, the disk-shaft assembly was designed to have a very low mass of approximately 200 g. (Fig. 7).

By reducing the mass of the disk-shaft assembly it was theoretically possible to raise the axial and lateral modes to around $600 \mathrm{~Hz}$ and $1 \mathrm{kHz}$ respectively which produces a significant interference-free region to observe a large portion of the bearing harmonics. To retain a constant rolling element running track, a soft preload was applied through a spring spacer setup. This was chosen during the design phase; different spacer thicknesses could be used to test multiple preloads to determine the most effective. Although 
Fig. 6 Section view of the KBT

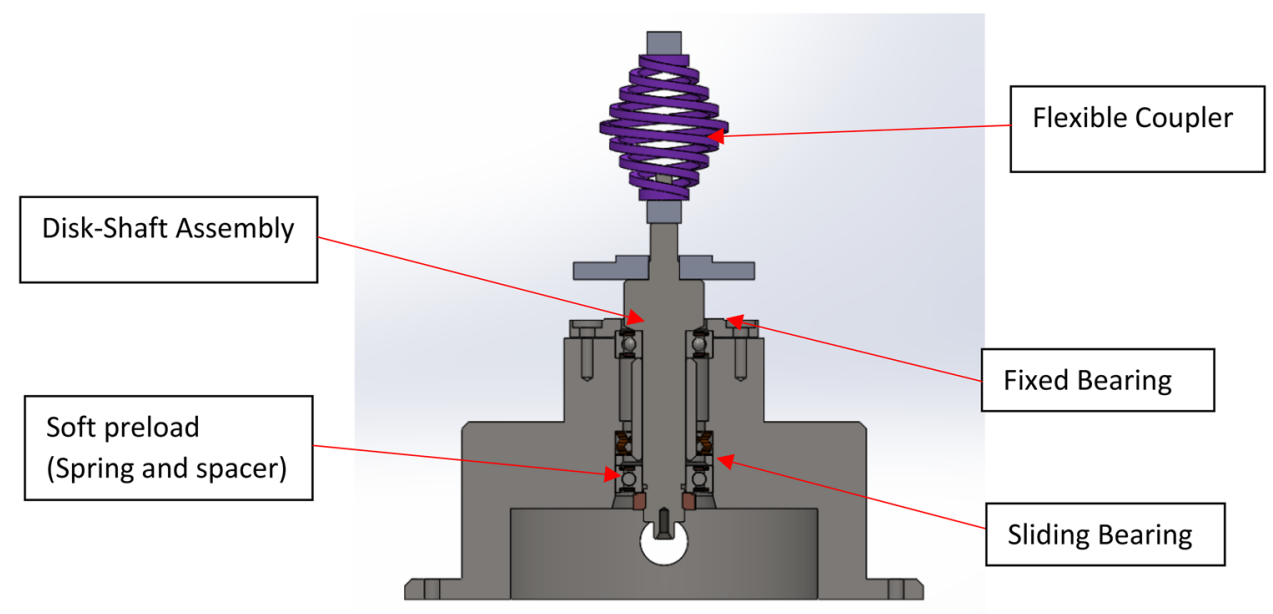

Fig. 7 Flexible Coupler and FEA modal analysis results

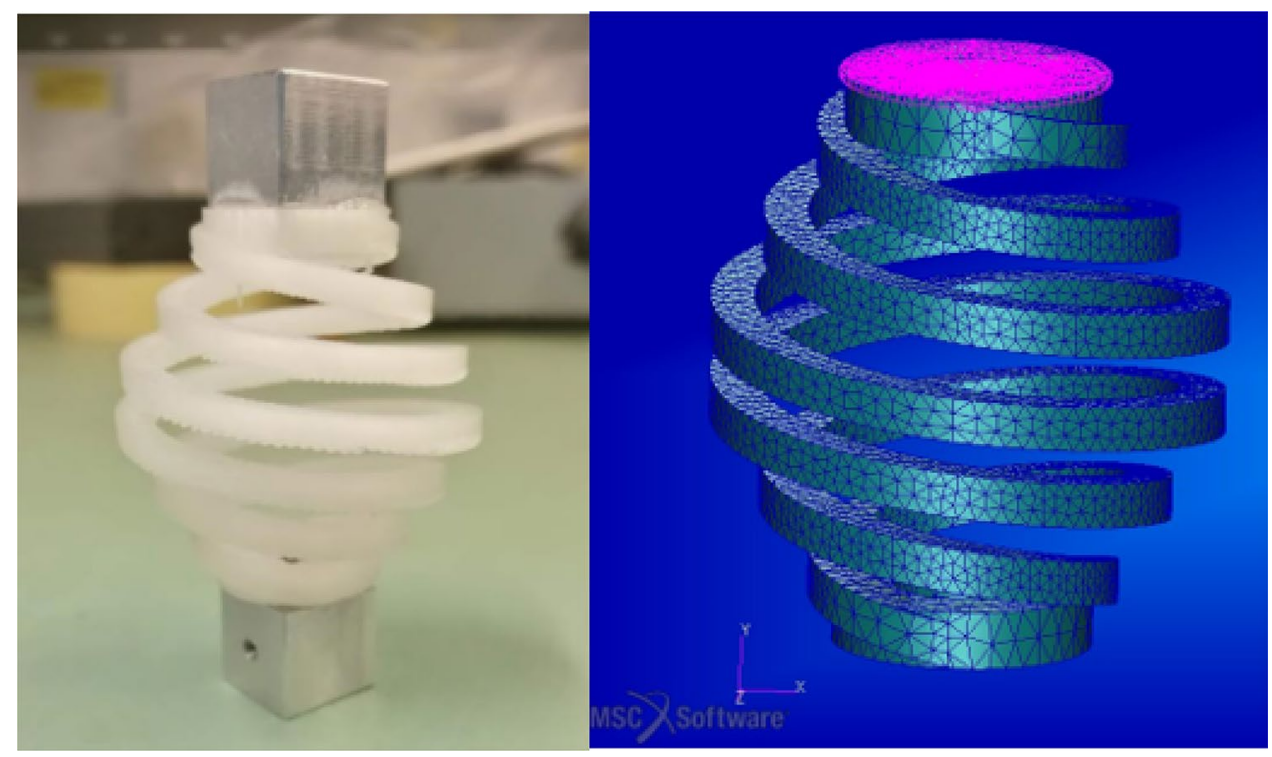

Table 4 Proposed preloads their reasoning and the measured preload

\begin{tabular}{llll}
\hline $\begin{array}{l}\text { Spring load/slid- } \\
\text { ing bearing (N) }\end{array}$ & $\begin{array}{l}\text { Estimated fixed } \\
\text { bearing (N) }\end{array}$ & Reasoning & $\begin{array}{l}\text { Meas- } \\
\text { ured } \\
\text { preload }\end{array}$ \\
\hline 33 & 35 & Minimum flight & 30 \\
40 & 42 & Maximum flight & 38 \\
60 & 62 & Intermediate & 51 \\
80 & 82 & Double flight & 72 \\
\hline
\end{tabular}

multiple preloads were tested, a load of $40 \mathrm{~N}$ was chosen as it most represented the preloads used in a flight-ready RWA; Table 4 shows a list of the preloads tested during the design phase and the actual measured preloads.
To then remove the motor disturbances, the motor was removed from within the housing and placed upon a secondary structure above. It was then connected to the top of the disk through a low stiffness flexible coupler which acted as a low pass filter heavily dampening any significant motor disturbances.

To ensure that flexible coupler would act as a low pass filter it would need incredibly low natural frequencies. A desired limit of $10 \mathrm{~Hz}$ was chosen, and multiple designs and materials were investigated however it was decided that a helix spring style design made from additively manufactured PLA would be the best option. Modal analysis was done using an applied load of $300 \mathrm{~g}$ as that is the mass of the motor. From the results, it showed that natural frequencies in the axial and lateral directions should be approximately 5.86 and $3.88 \mathrm{~Hz}$, respectively. The 
Table 5 stiffness and natural frequencies assuming a $300 \mathrm{~g}$ load

\begin{tabular}{ll}
\hline Material & PLA \\
\hline Axial stiffness & $410 \mathrm{~N} / \mathrm{m}$ \\
Lateral stiffness & $180 \mathrm{~N} / \mathrm{m}$ \\
Axial natural frequency & $5.86 \mathrm{~Hz}$ \\
Lateral natural frequency & $3.88 \mathrm{~Hz}$ \\
\hline
\end{tabular}

simulated natural frequencies and stiffness' can be seen in Table 5

\subsection{Measurement and isolation system}

To record the microvibrations the test setup was bolted to a Kistler table as shown in Fig. 8, which has 4 triaxial force transducers placed in the corners to measure the disturbances in the lateral and axial directions. The resultant signal from the transducers was recorded using a sampling rate of $2560 \mathrm{~Hz}$. The Kistler table is situated on an isolation system comprising of a large granite block and pneumatic isolators. This ensures that minimal background noise is picked up by the force transducers. Figure 9 shows the full setup of the kistler table and isolation system. To determine the overall response in each cartesian axis, a sum of each relevant transducer output is taken.

To observe the evolution of the bearing harmonics with respect to the rotational speed, the KBT was spun from 0 to 6000 RPM accelerating using a ramp function of 3 RPM per second. Although the motor used can record the speed, an encoder was attached to the bottom of the disk-shaft assembly so that the actual rotational speed could be recorded. The
Fig. 8 Close up image showing the experimental test setup

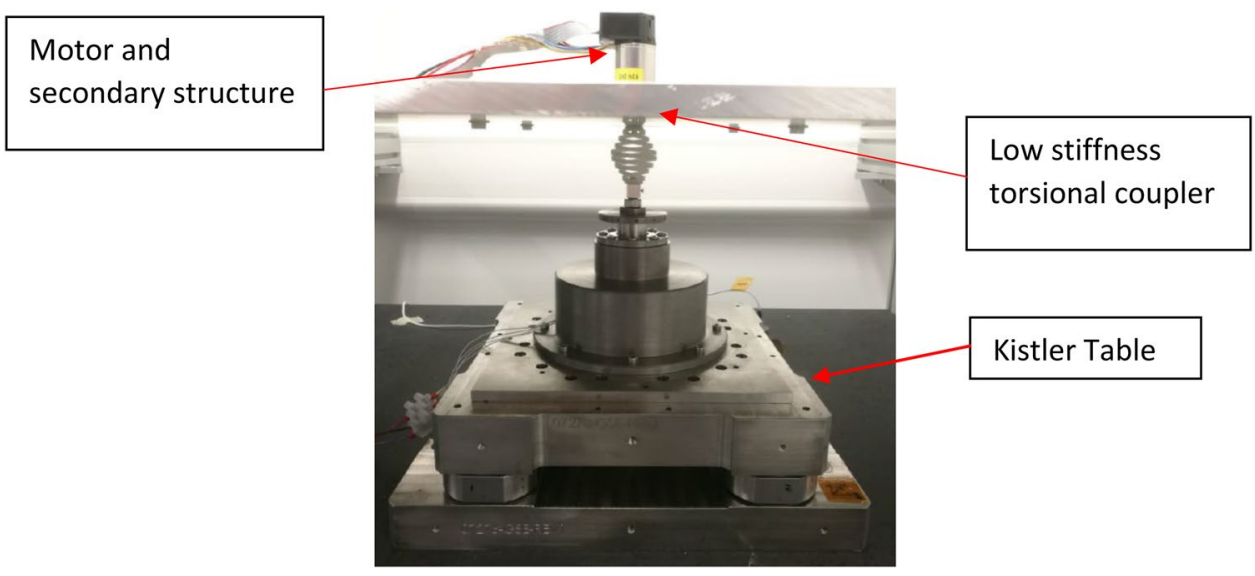

Fig. 9 Isolation system used to remove any external noise from the measured results

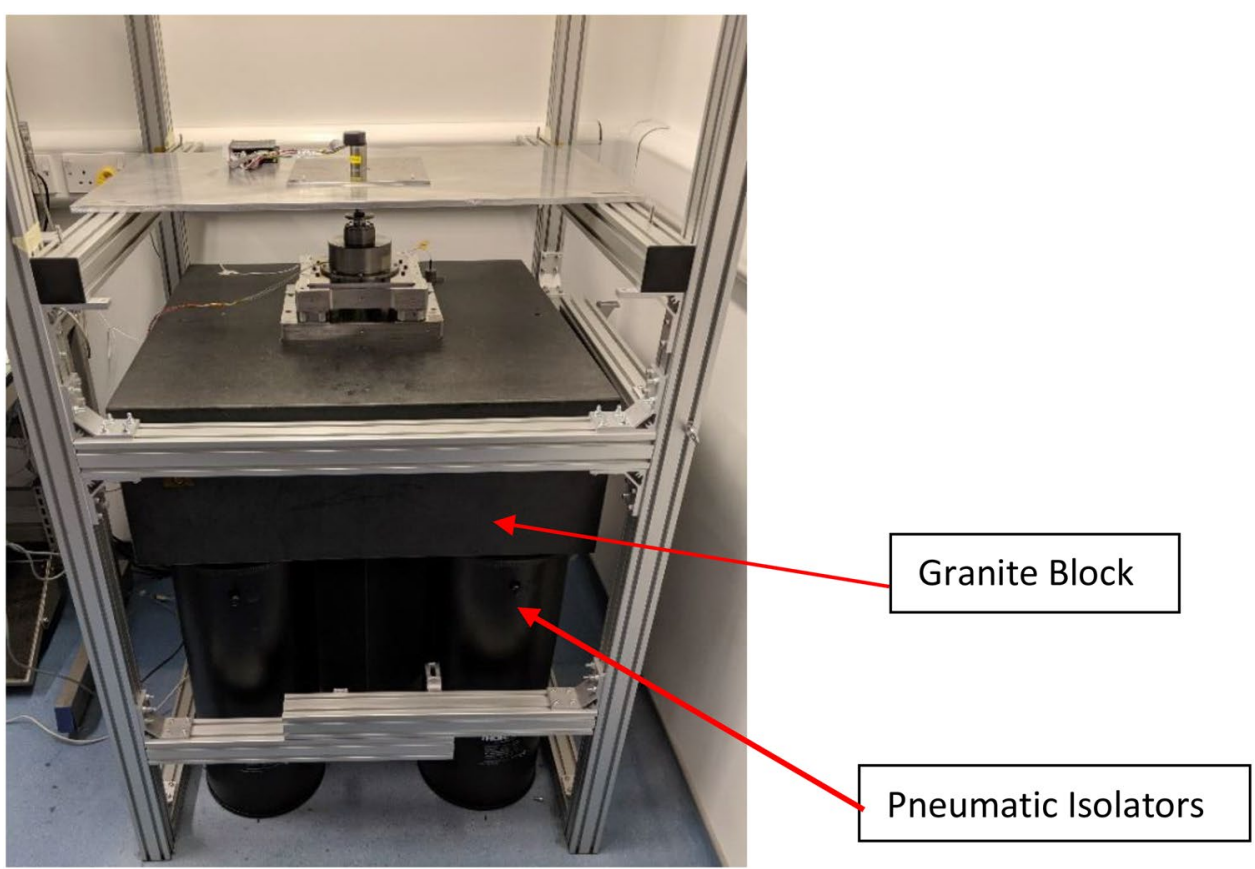




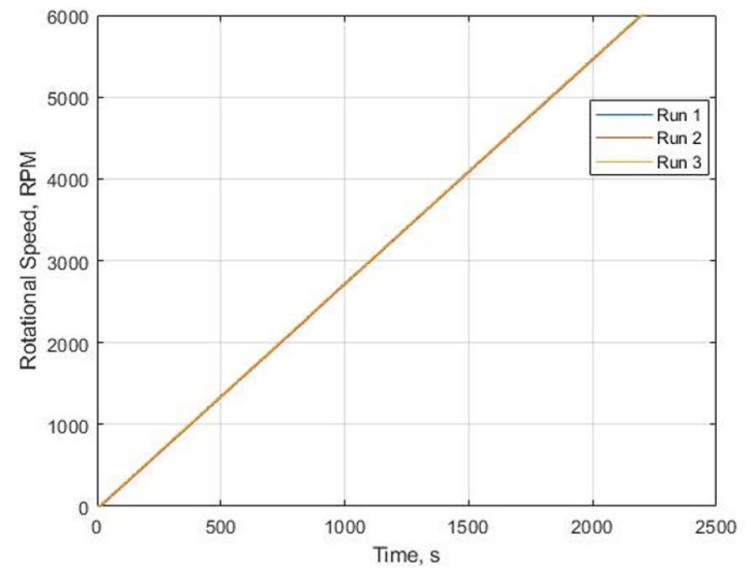

(a)

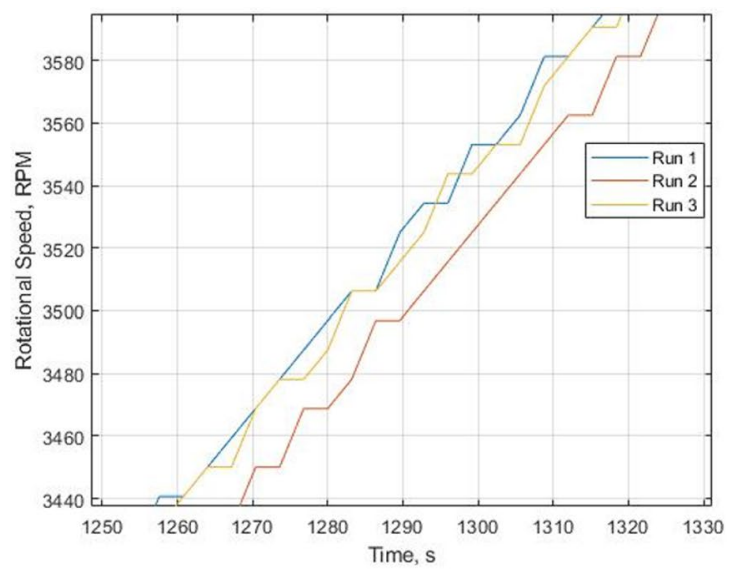

(b)

Fig. 10 Speed measurements of the KBT a Full-speed range, b Zoomed in section showing fluctuations

Table 6 Average acceleration per second:

\begin{tabular}{llll}
\hline Run & \multicolumn{3}{l}{$\begin{array}{l}\text { Average Acceleration } \\
\text { (RPM/s) }\end{array}$} \\
\cline { 2 - 4 } & AB & CD & EF \\
\hline $\mathrm{a}$ & 2.775 & 2.782 & 2.781 \\
$\mathrm{~b}$ & 2.777 & 2.786 & 2.782 \\
$\mathrm{c}$ & 2.777 & 2.786 & 2.782 \\
\hline
\end{tabular}

results of three runs can be seen in Fig. 10 and the average accelerations for each of the three bearing pairs are noted in Table 6.

\section{Results}

The aim of this section is to display a portion of the results of the microvibration tests carried out using the KBT. Initially, some results are used with the purpose of validating the KBT as a test rig which can simulate the motion of an RWA but produces an interference-free region so that the evolution of the bearing disturbances can be observed. To determine whether the KBT can be deemed successful some criteria was set, which were:

1. Natural frequencies of in family assemblies must remain within $\pm 10 \%$ between runs

2. There must be an interference-free region large enough to encompass the full list of fundamental harmonics calculated in Table 3 for a wheel speed range of 0-6000 RPM in the radial direction.

To analyse and make sense of the results the time domain data was subjected to a fast Fourier transform (FFT) to convert into the frequency domain. This was achieved by separating the time domain data into blocks of $3.2 \mathrm{~s}$ where an FFT was carried out on each block using the in-built MATLAB functions. For each block, a speed was assigned by taking the average of all the recorded speed values that were collected during that 3.2 s. Stitching the frequency domain blocks together for each recorded wheel speed, Campbell diagrams can be created. These are used as a tool to inspect the results at a "top level" as although they do not provide a quantitative observation, they can provide insight into the location of the dominant modes present in the system. Following the validation of the KBT, results of two test campaigns are presented one to observe the effects of reassembly and the effects of the static unbalance. In these tests, a focus will be on the harmonic engine orders which can be associated with the analytically determined shown in Table 3.

When comparing the results at a top-level two approaches are taken, an inspection of the Campbell diagrams to observe the structural modes and any other interesting phenomena. However, to provide a quantitative comparison of the whole systems response, a method was used which approximates the volume under the Campbell diagram using the force as the 3rd axis produces a number than can be used to represent the total disturbance the system produces during the test.

To achieve this, after the raw time data was converted into the frequency domain a trapezoidal function was carried out for each frequency within the range of interest $(0-600 \mathrm{~Hz})$ and then summed together. Whilst there are many methods to compare the response of a system this was thought to be useful as it provided a value which can be used to easily compare other cases. A similar approach can be taken when carrying out a quantitative comparison of the harmonics where the area under the curve is taken. However, when focusing on specific engine orders the area 
Fig. 11 Harmonic quantitative method

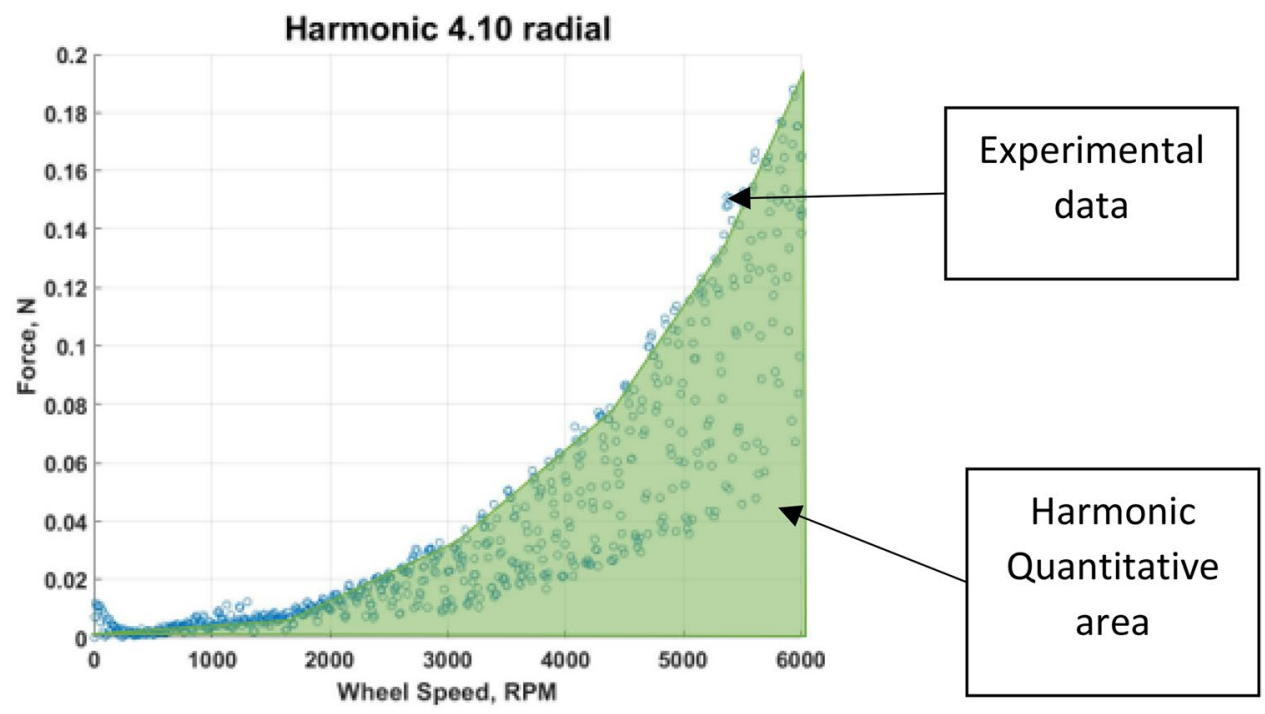

is taken after the data has been converted into the order domain. A graphical representation of the harmonic quantitative method can be seen in Fig. 11.

\subsection{KBT validation}

To ensure that the KBT provides the desired effects, a comparison of Campbell diagrams between a SSW-110 RWA, the KBT with a SSW-110 inspired $2 \mathrm{~kg}$ disk and KBT with the $200 \mathrm{~g}$, showing the vibration signature along one of the radial directions can be seen in Fig. 12. These were created by performing a ramp acceleration from rest to 6000 RPM. It can be seen that all the key features are in all three cases, however with the desired effect that the structural modes are significantly raised with the lightweight disk. In the SSW-110 diagram provided by SSTL it can be seen that the lateral mode is originally around $600 \mathrm{~Hz}$ whereas it is above $1000 \mathrm{~Hz}$ in the KBT. Similarly, in the axial direction the axial mode for in family assemblies has been increased from approximately $250 \mathrm{~Hz}$ to $520 \mathrm{~Hz} \pm 10 \%$. Whilst this is not truly the full desired range in the axial direction it does provides a large enough frequency range to cover engine order 5.93 up-5000 RPM without any interference which will be enough to show the amplitude evolution. (Fig. 13).

Whilst a Campbell diagram created in the Frequency domain is useful for identifying certain features like the system's resonance modes, non-integer harmonic orders can be more difficult to identify. An alternative method would be by converting the frequency domain into the order domain by dividing the data point frequency by the corresponding wheel speed, this essentially rotates the radial lines representing the harmonics creating a Campbell diagram which is easier to read. An example of a Campbell diagram created using the order domain data can be seen in Fig. 14. Whilst it is easier to identify and isolate specific disturbances, the drawback to using the order domain is the lack of precision at lower wheel speeds due to the potential overlap between some frequencies which can be caused by the resolution.

Although the main desired feature of the KBT was to design a test rig with a large interference-free region, the purpose behind this was to understand how harmonics evolve with the wheel speed to help develop an accurate model to predict the bearing disturbance amplitudes. Isolating data points which could belong to the orders in Table 3 calculated using the equations shown in Table 1 are then shown in Fig. 15. Whilst order 5.93 does appear to show some interference from a resonance spike at approximately 5500 RPM in the axial direction, it can be seen that the more dominant engine orders follow a parabolic function. At 5500 RPM this disturbance would have a frequency of $543 \mathrm{~Hz}$, so this spike is likely to come from the axial mode.

\subsection{Effect of reassembly}

An important concept to understand when carrying out any tests to determining the effects of parameters that require modifications to a system is the variability between build assemblies. By understanding this, it will provide an insight into whether the change in disturbance is caused by reassembling the system or the parameter itself. Before embarking on adjusting any other parameter, 3 sets of nominally identical bespoke SR8 bearings each undertook 10 assemblies following an assembly procedure fitting of industrial standards. As each test was carried out using nominally identical builds it was expected with some degree of confidence that each set of results should appear almost identical. This was not the case, whilst the in family assembly resonance frequencies stayed within $10 \%$ of the average and the bearing disturbances retained the same harmonic orders, the harmonic 


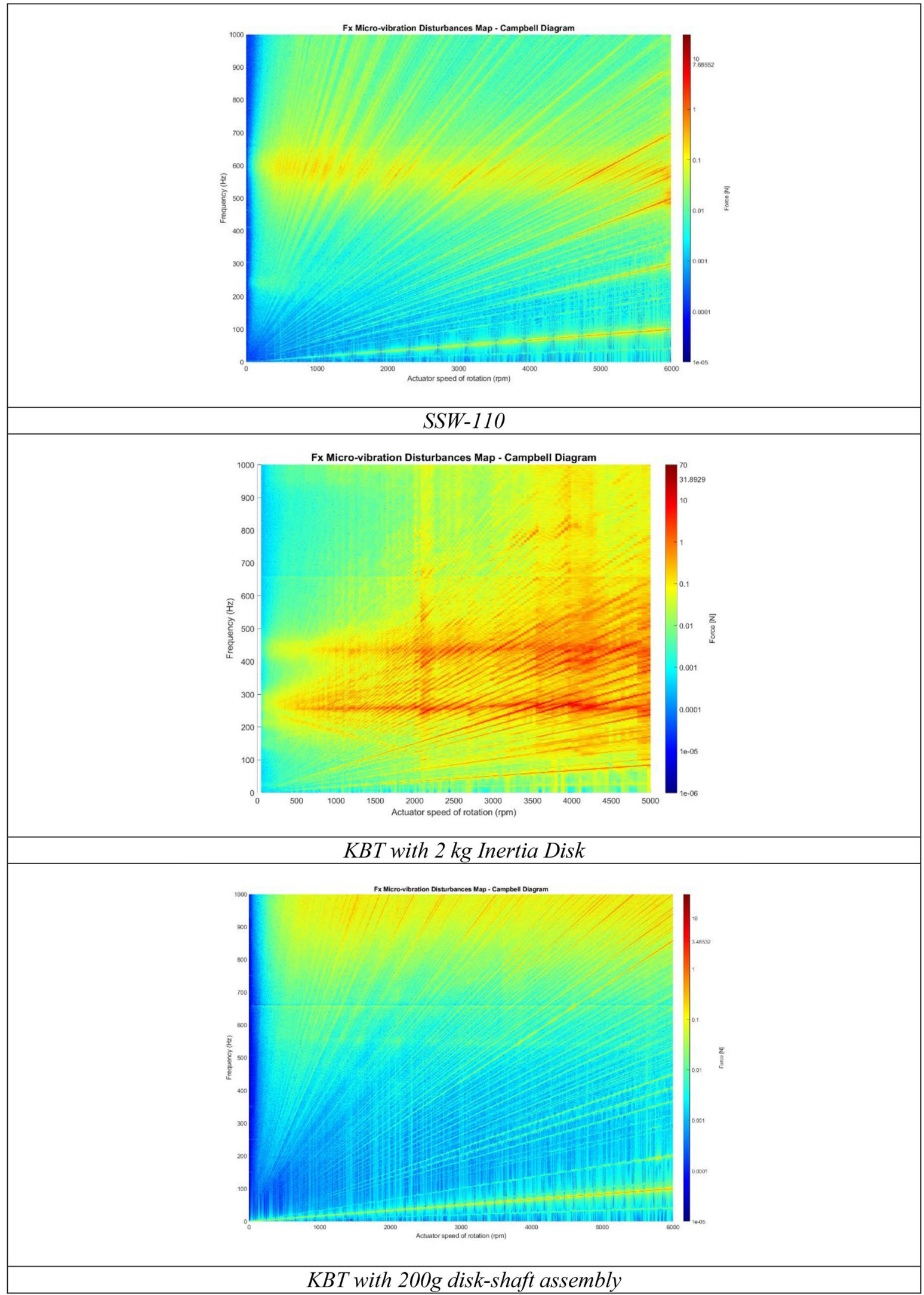

Fig. 12 Campbell diagrams comparing the microvibration signature of an SSW-110 RWA and the KBT in the radial directions 
Fig. 13 Campbell diagram of $\mathrm{KBT}$ in the axial direction

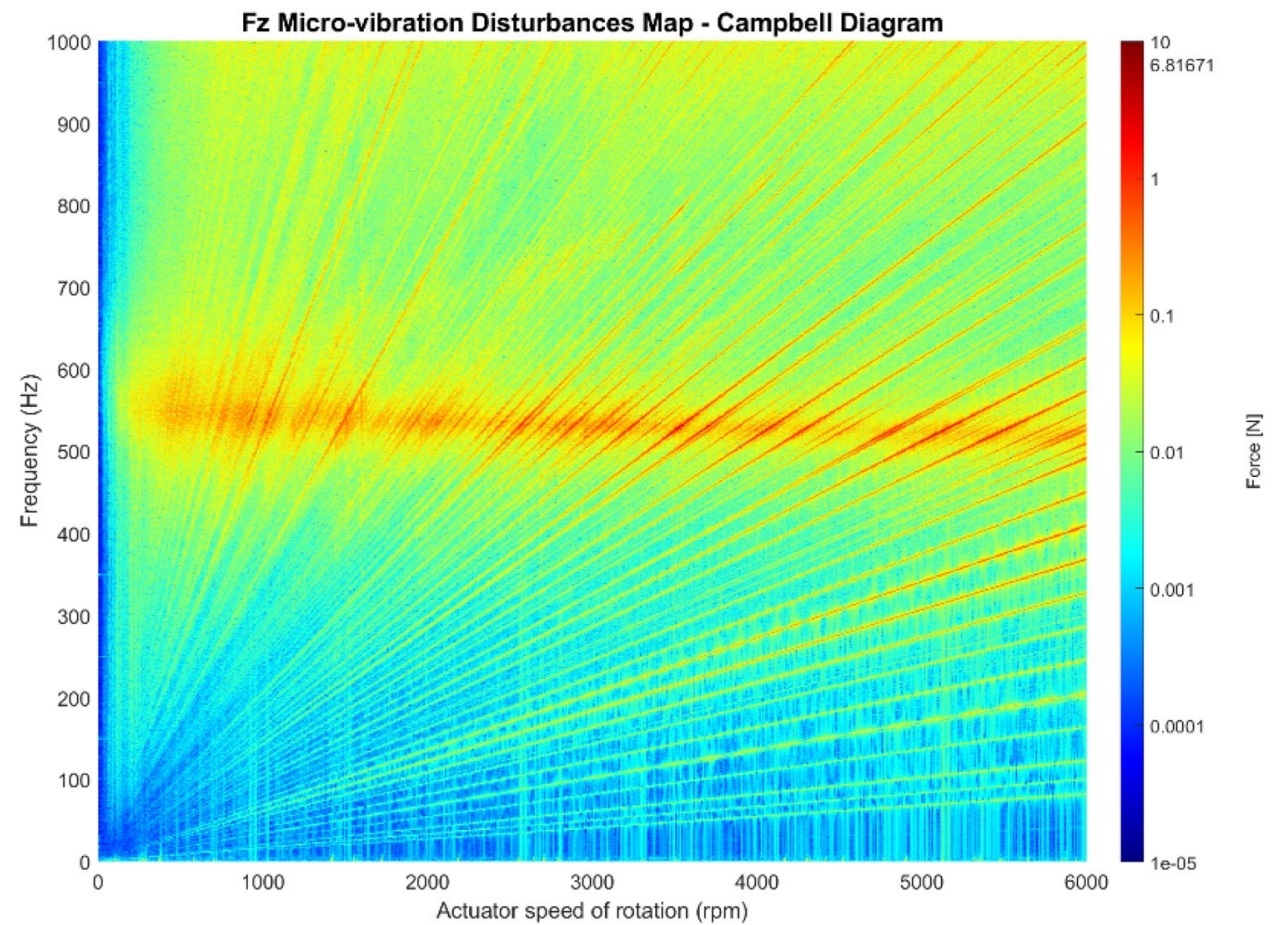

Fig. 14 Order domain Campbell diagram

Fig. 15 Harmonic development with wheel rotational speed
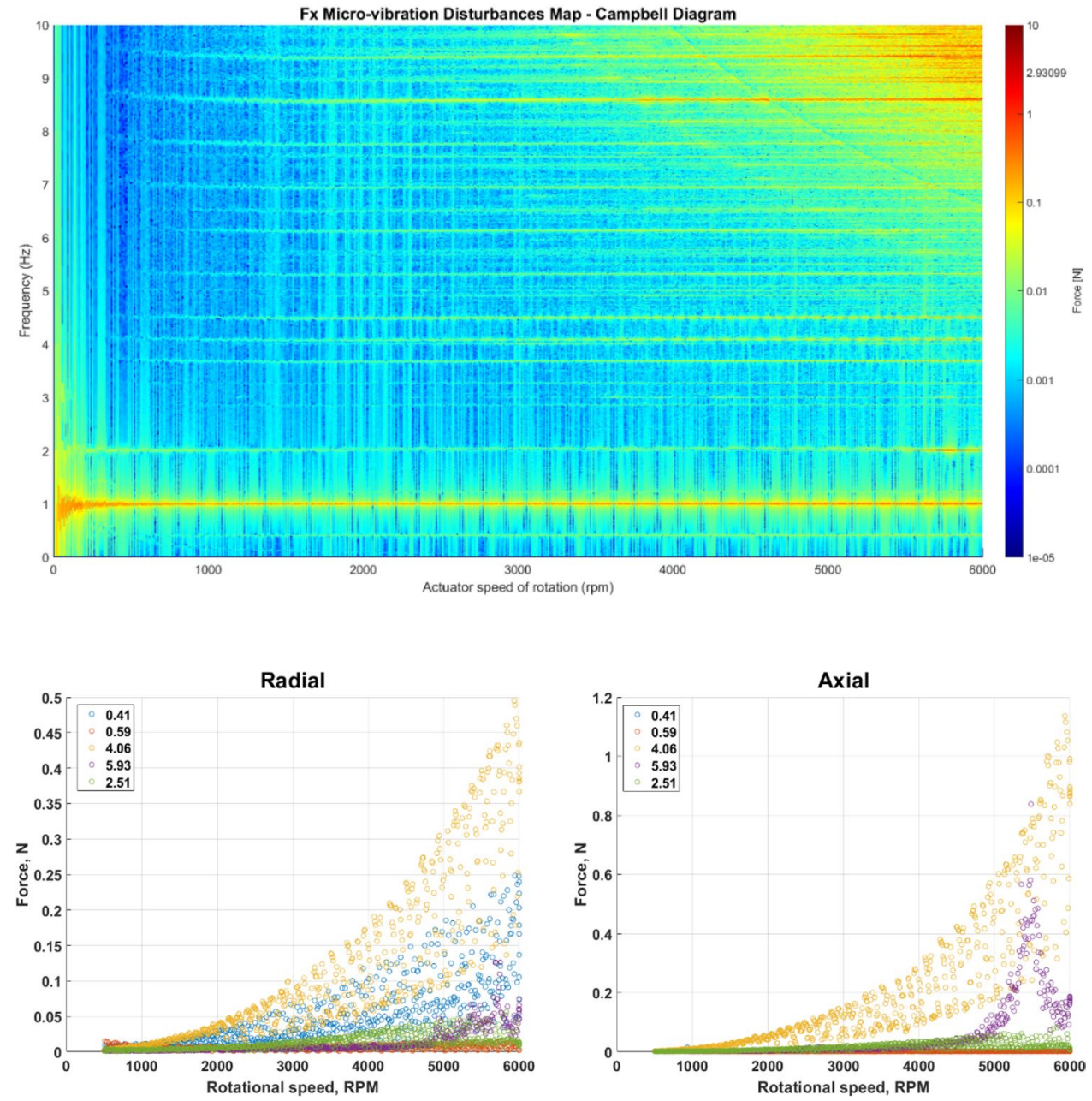
Fig. 16 Total disturbance for each build assembly

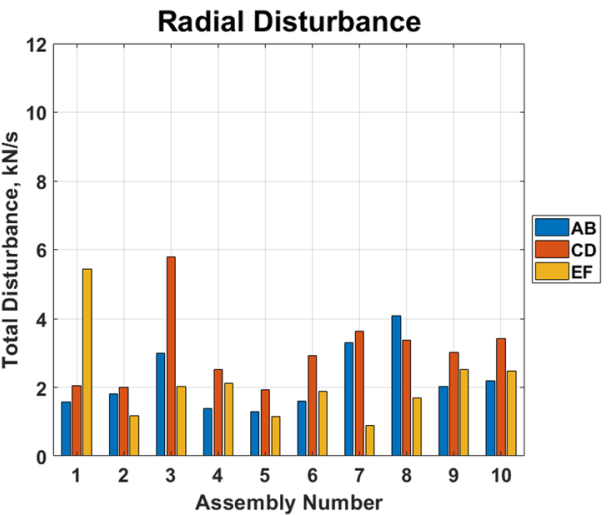

(a)

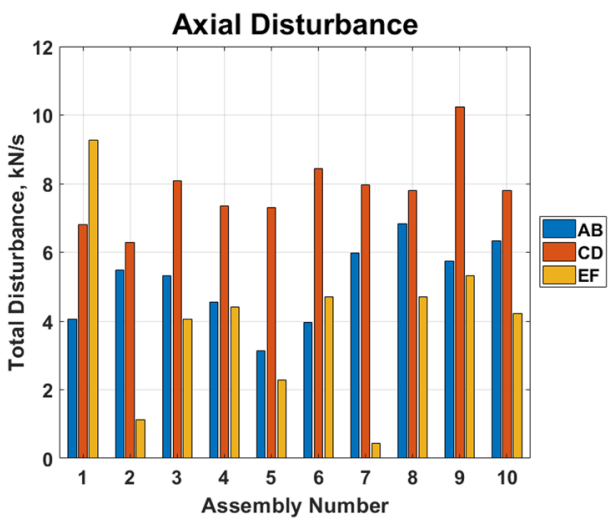

(b)
Table 7 Mean and standard deviation of total disturbances seen between each build assembly

\begin{tabular}{llllll}
\hline Bearing Pair & \multicolumn{2}{l}{ Mean disturbances $(\mathrm{kN} / \mathrm{s})$} & & \multicolumn{2}{l}{$\begin{array}{l}\text { Standard deviation } \\
(\mathrm{kN} / \mathrm{s})\end{array}$} \\
\cline { 2 - 3 } \cline { 6 - 6 } \cline { 6 - 6 } & Radial & Axial & & Radial & Axial \\
\hline $\mathrm{AB}$ & 2.23 & 5.14 & & 0.93 & 1.17 \\
$\mathrm{CD}$ & 3.06 & 7.81 & & 1.14 & 1.07 \\
$\mathrm{EF}$ & 2.14 & 4.05 & & 1.29 & 2.46 \\
\hline
\end{tabular}

amplitudes received significant variation. As there are many different disturbances that occur within the KBT or an RWA, it is important to consider many different aspects when comparing different cases. For this study, an approach was taken in which the system was observed at a top-level and then narrowed down to focus on specific disturbances.

\subsubsection{Top level}

There are many different approaches when analysing microvibrations, however many of these focus on specific features such as the harmonics or structural modes and as a result they do not provide a quantitative comparison of the whole disturbance. To observe the system response at a top-level two approaches were chosen, the first was a visual inspection of the Campbell plots seen previously in Figs. 12 and 13 which were inspections to ensure all the key features such as the structural modes were similar meaning that the measurable natural frequencies remain in approximately the same frequency $\pm 10 \%$. To produce a quantitative comparison at a very top level, approximating the volume under a waterfall plot for a given frequency range was chosen as it quantifies the total disturbances and provides a rough overview for all disturbance types. Whilst this method does not produce any information about specifics it does provide a view of how the whole system reacts. This method can be seen in Fig. 16 which compares the total disturbances seen across each build

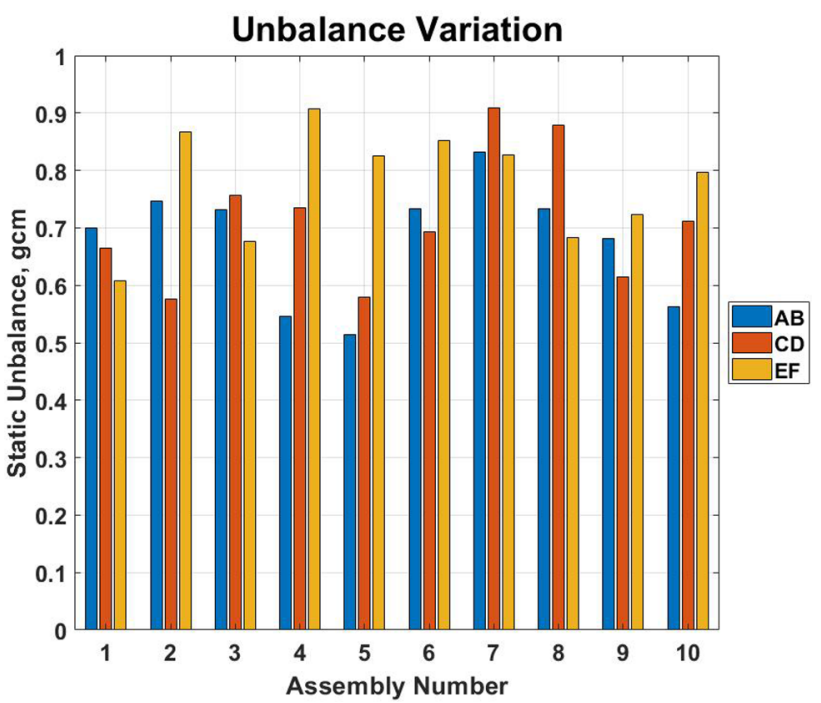

Fig. 17 Static unbalance comparison between assemblies

for the three sets of bearings. The mean and standard deviation of each bearing pair is then shown in Table 7. At a toplevel, there is an unexpected but clear variation between the total disturbances in each build assembly.

Whilst nominally identical RWAs should produce similar disturbances when using the same equipment and assembly procedure, however, this was not the case as when comparing the assemblies at a top-level the total disturbances observed appeared to have significant variation. Although, a trend was noticed in that bearings that were subjected to prior environmental testing appeared on average to be noisier. Inspecting the unbalance to check for potential issues during the build process which are then plotted in Fig. 17, with the mean and standard deviation noted in Table 8 although there is some change the variation seems to be somewhat consistent. This was expected, the disk-shaft assembly can be considered a single unit as the disk is attached to the shaft using an interference 
Table 8 Mean and standard deviation of the static unbalance between assemblies

\begin{tabular}{lll}
\hline Bearing pair & Mean unbalance $(\mathrm{gcm})$ & $\begin{array}{l}\text { Standard } \\
\text { deviation } \\
(\mathrm{gcm})\end{array}$ \\
\hline $\mathrm{AB}$ & 0.6782 & 0.1029 \\
$\mathrm{CD}$ & 0.7120 & 0.1141 \\
$\mathrm{EF}$ & 0.7765 & 0.0980 \\
$\mathrm{All}$ & 0.7222 & 0.1097 \\
\hline
\end{tabular}

fit. There it can be assumed that any change in unbalance can be accredited to the clearance in the bearings. From the maximum radial clearance provided in Table 2 and taking the mass of the disk-shaft assembly as $200 \mathrm{~g}$ the maximum change in unbalance would be $0.4 \mathrm{gcm}$. From this and the values seen in Table 8 can be considered reasonable. Another top-level check for the assembly is the location of the structural modes, although it was hard to judge the natural frequencies in the radial direction due to them being at the edge of the Kistler table's capabilities the axial mode appear to be somewhat consistent with $\mathrm{a} \pm 10 \%$ variation. From looking at the top level it is clear that additional investigations into specific smaller disturbances like the bearing harmonics are required.

To investigate the issue regarding the variation in the top-level disturbances a more detail investigation into the individual frequencies of interest must be made. A common approach to achieve this is to produce a force envelope in the frequency domain at specific wheel speeds. A force envelope produces an insight into the maximum and minimum outputs for each frequency a mechanism produces. As all the tests in this study are created through a ramp function encompassing all recorded speeds, an RMS was taken for each frequency within the range of interest which helped account for any random spikes. These force envelopes which are limited to the frequencies that do not see any interference from the axial mode can be seen in Fig. 18 and Fig. 19 where it can be
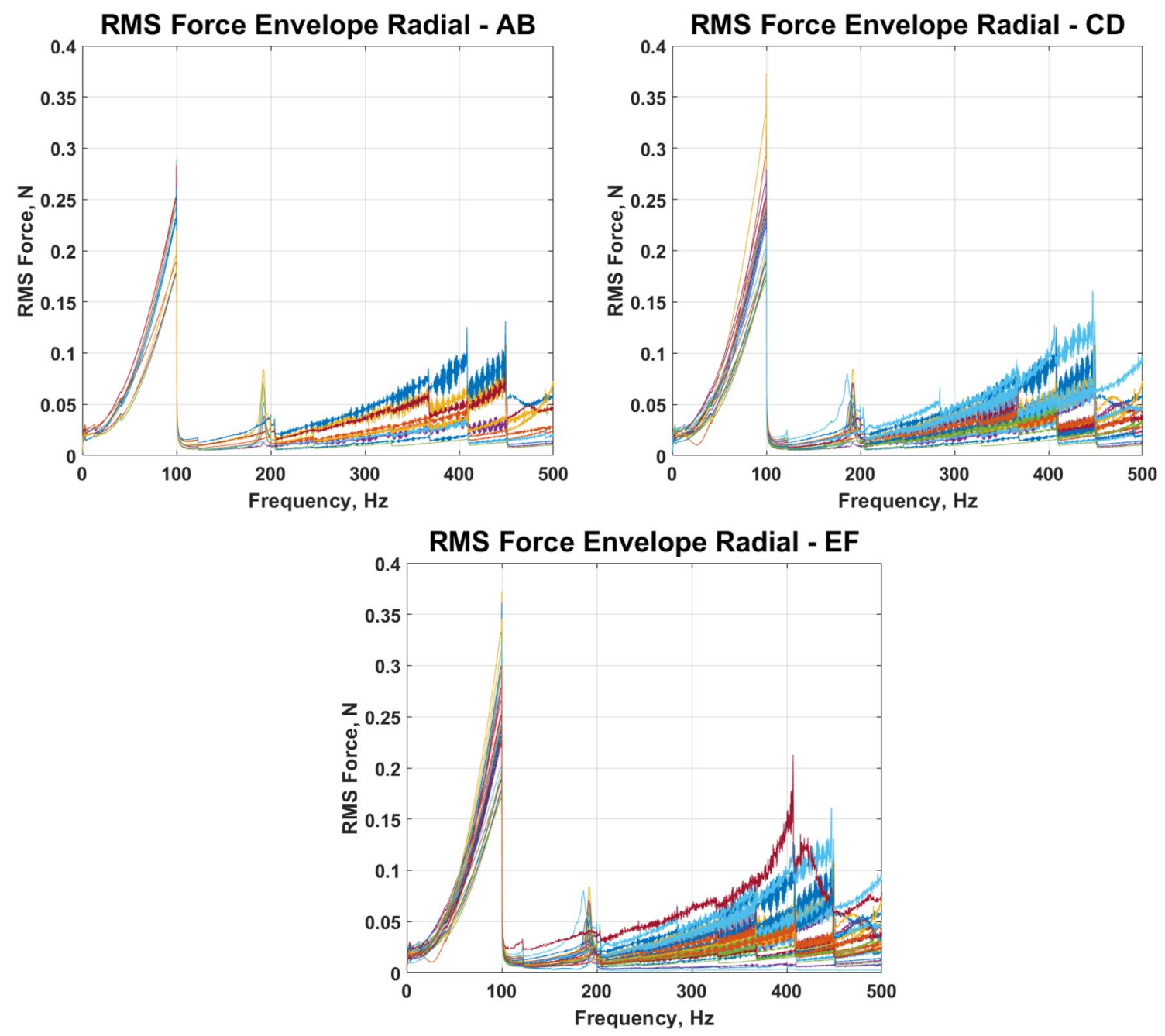

Fig. 18 RMS force envelope for each assembly in the radial direction 

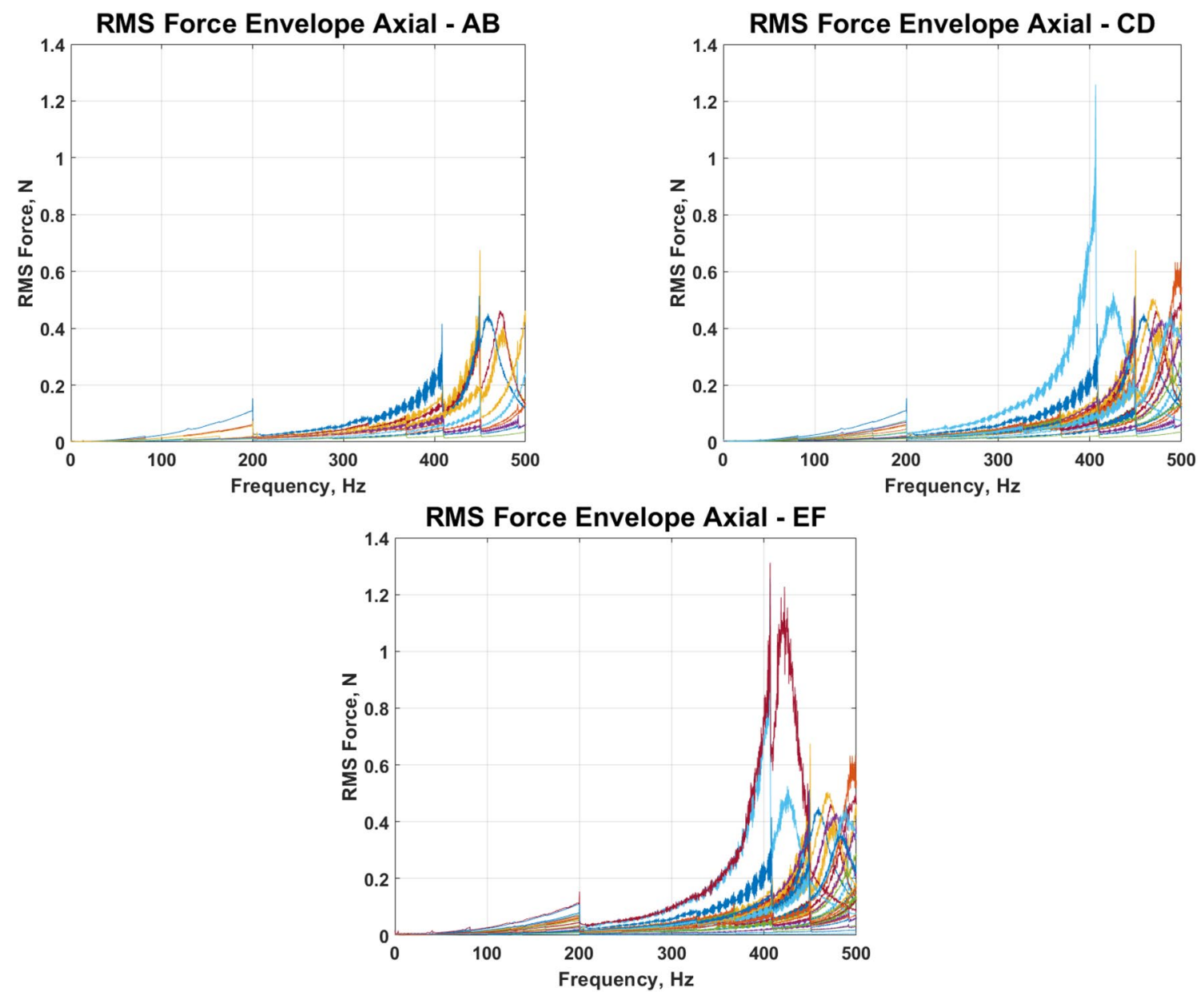

Fig. 19 RMS force envelope for each assembly in the axial direction

observed that there is consistently a dominant mode ending either at approximately 400 or $450 \mathrm{~Hz}$. From the analytical models it can assume that these modes are the FBOR (harmonic order 4.06) fundamental or a sideband created by interference with the FBOR and FCOR mode. Upon closer inspection of the harmonic disturbances that could be associated with FBOR, it was discovered that the variation seen between build assemblies is even more prominent.

\subsubsection{Harmonic level}

Whilst a force envelope is useful to determine the most dominant disturbances, as bearing disturbances develop with the rotational speed some tend to be overlooked. Instead by converting into the order domain and then measuring the area under each harmonic it is then possible to provide a quantitative approach to determine the most dominant harmonic disturbances. Figure 20 shows the top ten harmonics in the radial direction based on the total disturbance for each bearing set. As expected, the harmonics with the highest area are orders 1 , approximately 4.1 and 4.5 . Order 1 can be attributed to the unbalance whilst orders 4.1 and 4.5 based on the analytical models discussed in Table 1 can be associated with defects on the outer race. With order 4.1 being directly associated (FBOR) and order 4.5 is a sideband created by interference from disturbances originating on the inner race $(\mathrm{FBOR}+\mathrm{FCIR})$. The sideband disturbances are being noticed due to the origin of the disturbance and the location of the force transducers. As the transducers are located in the corners, anything that is observed in the centre of the Kistler table (location of the KBT) will cross paths with each. Engine order 2 is likely a second-order unbalance harmonic caused by some misalignment due to tolerances.

Similarly, variation to what was seen in both the total waterfall plot volume and force envelopes is clearly seen in each of the different harmonics. Whilst the change in the unbalance is expected due to handling, the change in the other harmonics is not expected. As the harmonic order numbers between assemblies can be considered consistent it can be assumed that based on the analytical models the contact angle is mostly constant between assemblies. 

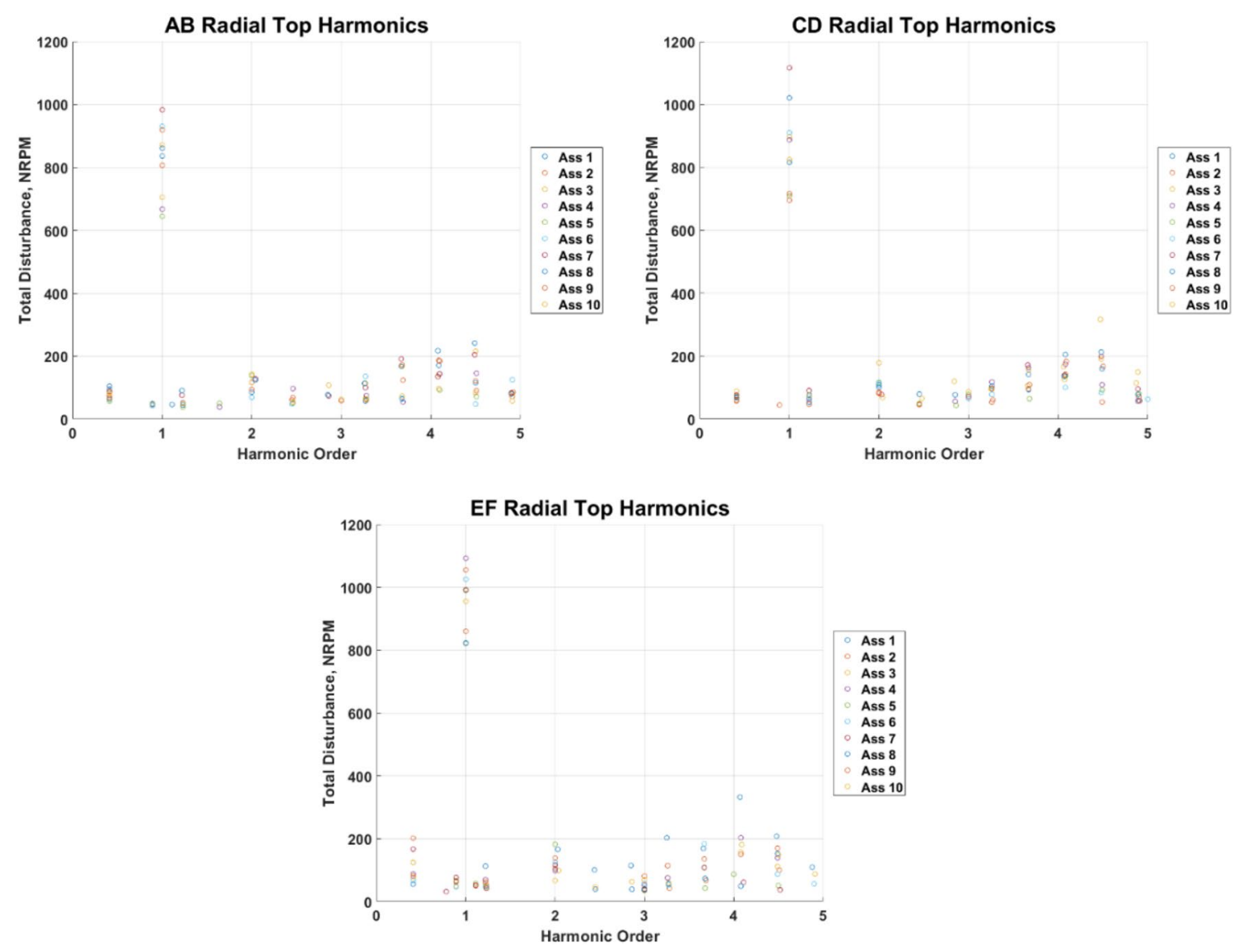

Fig. 20 Top ten harmonics

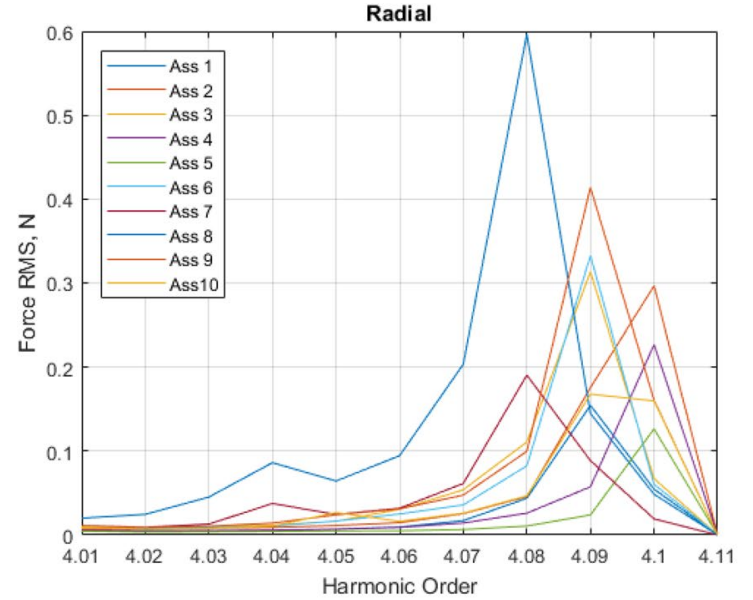

(a)

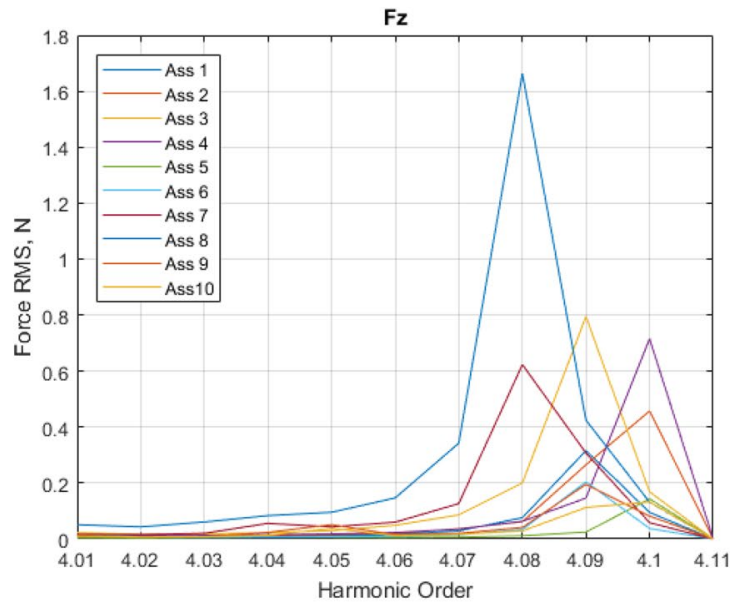

(b)

Fig. 21 Comparison of RMS values for each harmonic order that could be associated with an outer race defect detected in the a Radial direction, b Axial direction

A deeper and more detailed inspection of the harmonic disturbances can be seen in Figs. 21 and 22 which focus on the disturbances that could be associated with the FBOR analytical model. Figure 21 takes an RMS of the amplitudes across all wheel speeds for those that could be considered associated with a defect on the outer race. Whilst the analytical model predicts the disturbance should occur at order 4.06 based on the bearing parameters, the experimental results 


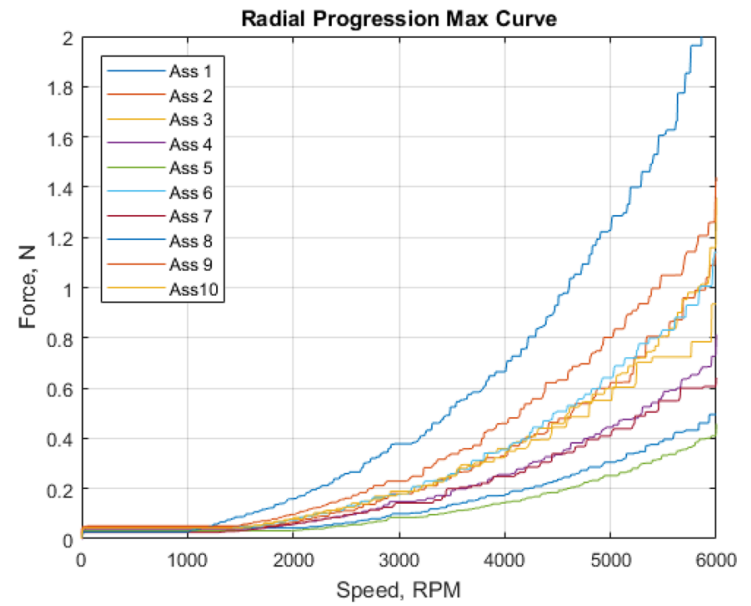

(a)

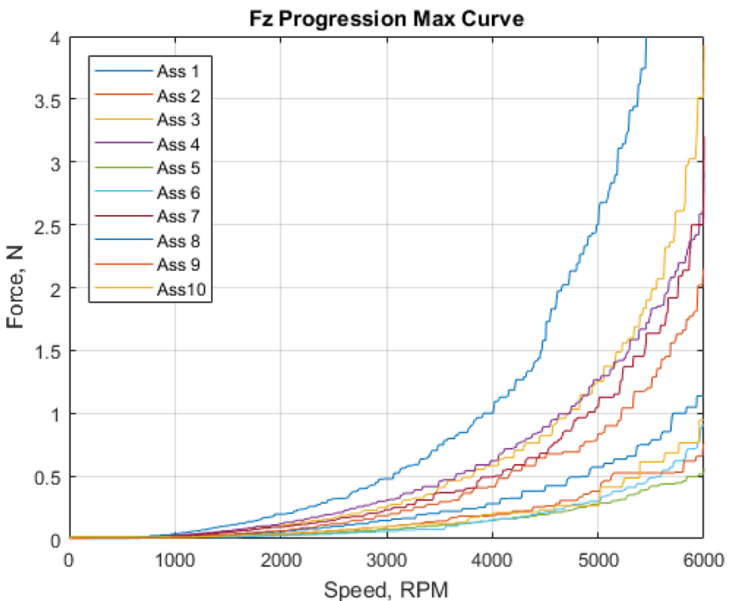

(b)

Fig. 22 FBOR disturbance evolution detected in the a Radial direction, b Axial direction

Table 9 Comparing the mean and standard deviation of the RMS response associated with the FBOR disturbance seen in the radial directions

\begin{tabular}{lll}
\hline Bearing Pair & FBOR RMS Mean $(\mathrm{N})$ & Standard deviation \\
\hline $\mathrm{AB}$ & 0.2819 & 0.1433 \\
$\mathrm{CD}$ & 0.2797 & 0.1327 \\
$\mathrm{EF}$ & 0.2372 & 0.2778 \\
\hline
\end{tabular}

show a disturbance between 4.08 and 4.10 which as that is only approximately $1 \%$ different it can be assumed that these are associated with the FBOR model. Figure 22 are then peak hold plots of the orders associated with the FBOR disturbance and showing the evolution of that harmonic. From these plots, it can clearly be seen that the harmonic follows a parabolic function, however, the coefficients seem to vary significantly between each assembly.

Like the top-level comparisons, the variation in amplitude is clearly shown in both the RMS and evolution plots. In an attempt to understand this variation, Table 9 displays the mean and standard deviation seen of the harmonics associated with the FBOR disturbance.

\subsection{Effect of the static unbalance}

Typically to test the parameters within an RWA the system must be reassembled to replace or adjust components however this proposes an issue, as seen with the test shown previously, there is a significant variation in results when reassembled. This begs the question of whether any change in amplitude is due to the parameter or a result of the variation caused by the assembly. However, as the static unbalance can be manipulated by adding additional mass through the

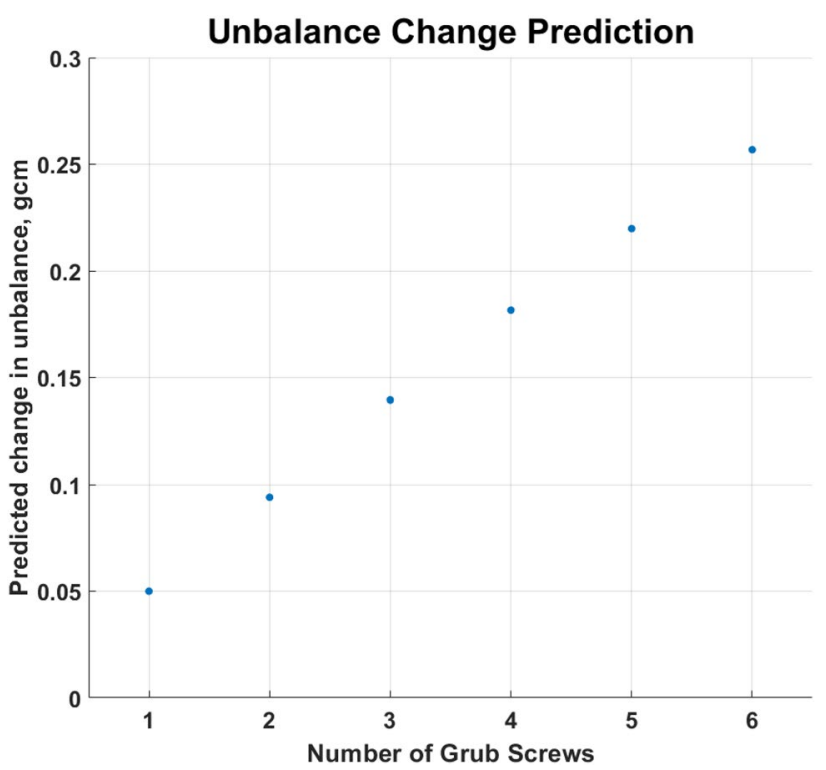

Fig. 23 Estimated change in static unbalance through the use of grub screws

use of grub screws, it is possible to change this parameter without any reassembly. These tests were done in two stages increasing then decreasing the static unbalance to produce a wide range. Predictions of how adding each grub screw will affect the unbalance was calculated using the product of the total mass of the grub screws and the radius of the centre of mass of the grubs when inserted in the inertia disk. It was assumed that two $\mathrm{M} 2 \times 3 \mathrm{~mm}$ screws could be inserted into each thread created onto the disk where each screw centre of mass was positioned directly in the centre. These predictions have been plotted in Fig. 23, where it can be seen that by 

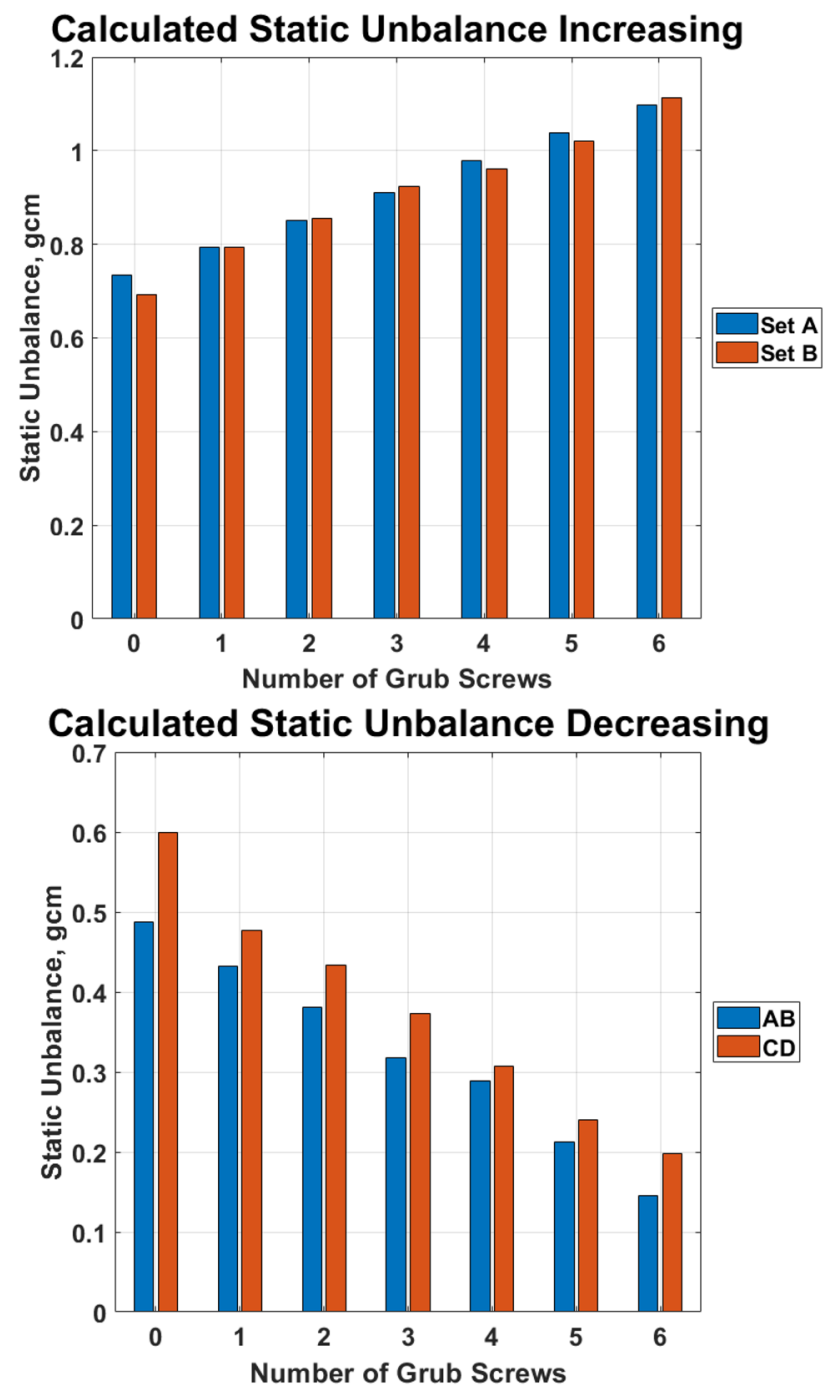

Fig. 24 Comparison of calculated static unbalance when adding grub screws to the inertia disk

adding 6 screws in the right place can increase or decrease the static unbalance value by approximately $0.26 \mathrm{gcm}$.

Unfortunately, the setup was reassembled between each stage, therefore, an initial run with no grub screws were done as a baseline to create comparisons for each scenario. Figure 24 shows the static unbalance of each case calculated from taking the average gradient of the first harmonic. Although they are not perfect the calculated unbalances follow a similar trend to what was expected based on the predicted changes.

Taking a similar approach when investigating the assembly variation, at a top-level there appears to be an almost negligible change. When increasing or decreasing the unbalance as seen in Fig. 25 and Fig. 26. As the unbalance can be considered a dominant disturbance it is also important to observe how at a top-level the system response is without the unbalance as this will provide an insight into how the unbalance influences the other harmonic disturbances and broadband noise. This can be seen in Fig. 25c, d. From these, the total disturbance does lower however the slight trend seen previously almost disappears.

Looking at the top ten harmonics shown in Figs. 27 and 28 , aside from the first order which changes as expected due to it being the unbalance disturbance, very little change is noticed within the others, Table 10 denotes the mean and standard deviation of the more dominant engine orders in the radial direction. Finally focusing on the FBOR disturbance it can be seen that in terms of the amplitude there is virtually no change when altering the static unbalance shown in Fig. 29.

\section{Conclusion}

By raising the structural modes out of the frequency range of interest through the use of a lightweight inertia disk and moving the motor outside to a secondary structure. A method to measure bearing microvibrations in a system that replicates a reaction wheel assembly without interference was shown. Using a slow ramp function, it was possible to measure the dominant frequencies for a wide range of rotational speeds. From comparing three geometrically identical bearings the following three conclusions can be made about the effects of reassembly and the static unbalance:

1. Whilst there are several disturbances that can be detected and associated with defects from the bearings used in this study, those originating from the interaction between the rolling elements and imperfections on the outer race seem to be the most dominant. This trend is also shown in the results displayed in [33] and predicted in [29]. The dominant modes can be seen by ramp disturbances ending at approximately $400 \mathrm{~Hz}$ and $450 \mathrm{~Hz}$ which represent the FBOR and FBOR + FCOR harmonics respectively. Similarly seen by the peaks at engine orders 4.08 and 4.51 when observed in the order domain.

2. When keeping the static unbalance within a reasonable range, the tests reported in this paper show no clear trend or negligible change in amplitude for any dominant bearing disturbance when manipulating the static unbalance. Other than the first order, which can be directly linked to the unbalance. When comparing the results at a top-level there is some trend which follows the unbalance change. However, after removing the data points associated with the unbalance there is very little change with suggests that the unbalance does not have a significant impact on the amplitudes of the broadband noise or bearing harmonics. However, this may be due to the level of force being transmitted by the unbalance relative to the force provided in the preload. 


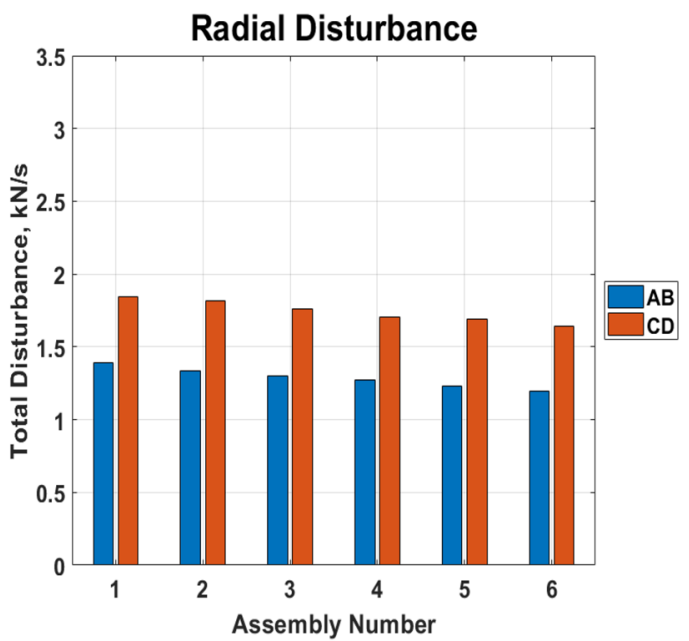

(a)

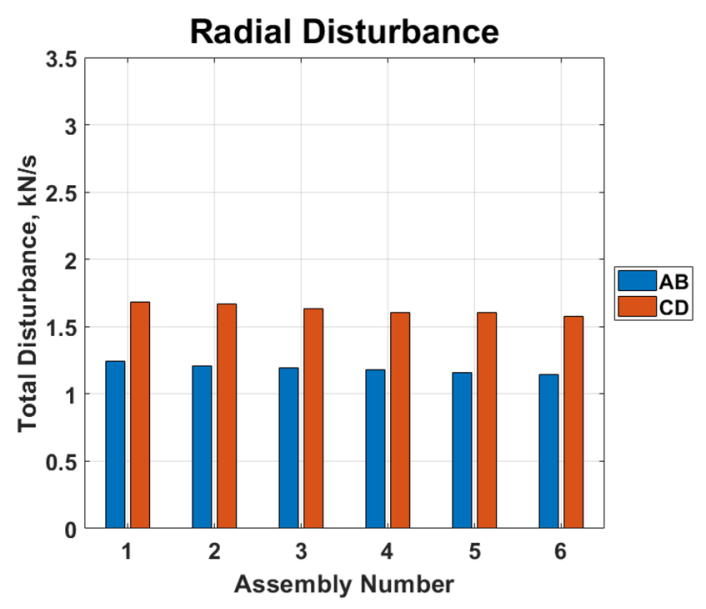

(c)

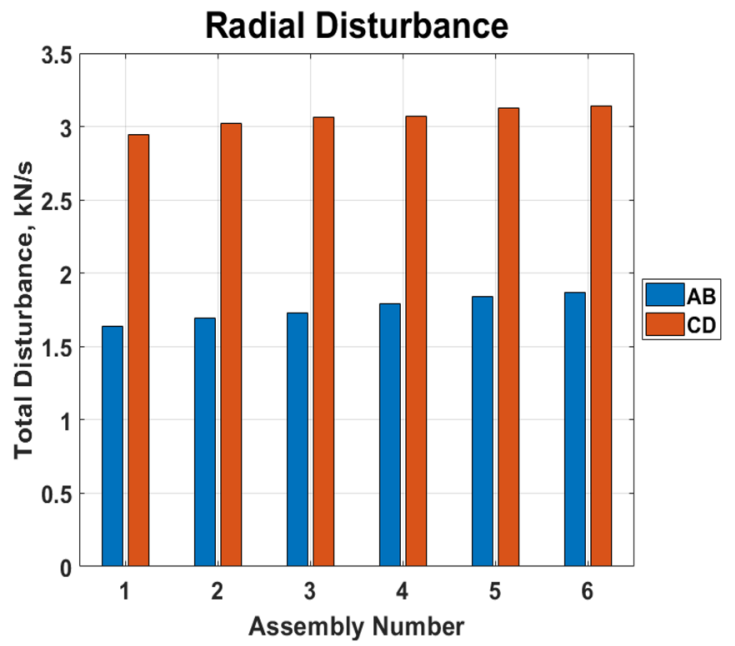

(b)

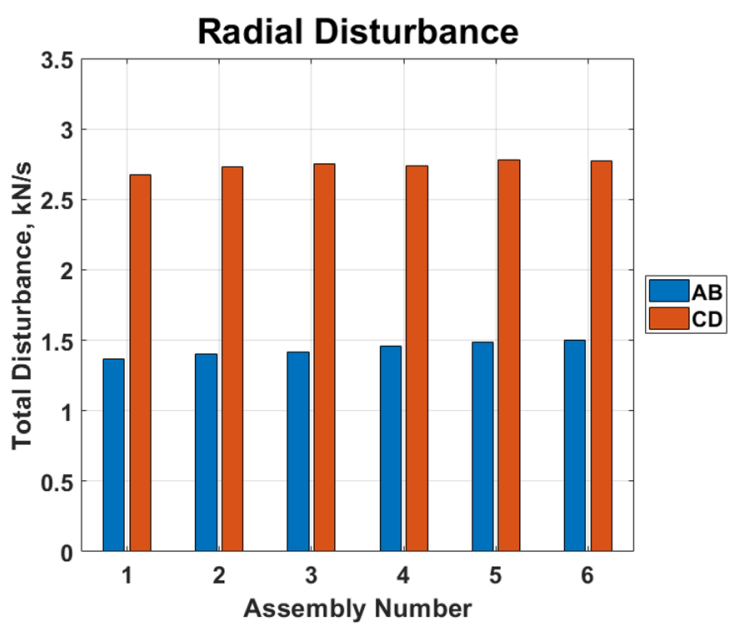

(d)

Fig. 25 Comparison of the total disturbance in the radial direction a Decreasing with unbalance $\mathbf{b}$ Increasing with unbalance $\mathbf{c}$ Decreasing without unbalance $\mathbf{d}$ Increasing without unbalance

Fig. 26 Comparison of the total disturbance in the axial direction a Decreasing b Increasing

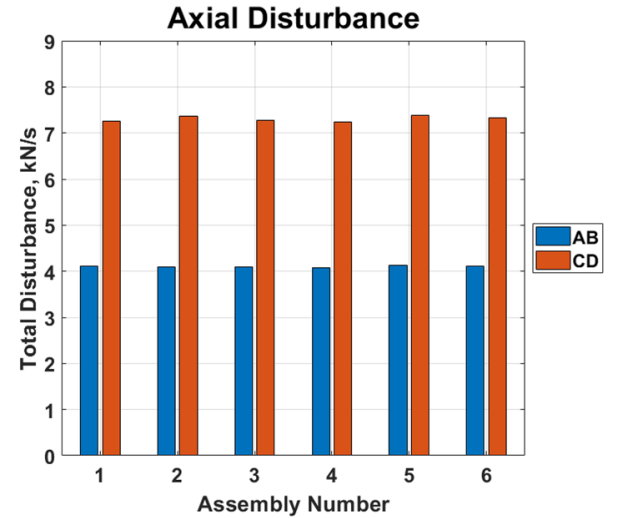

(a)

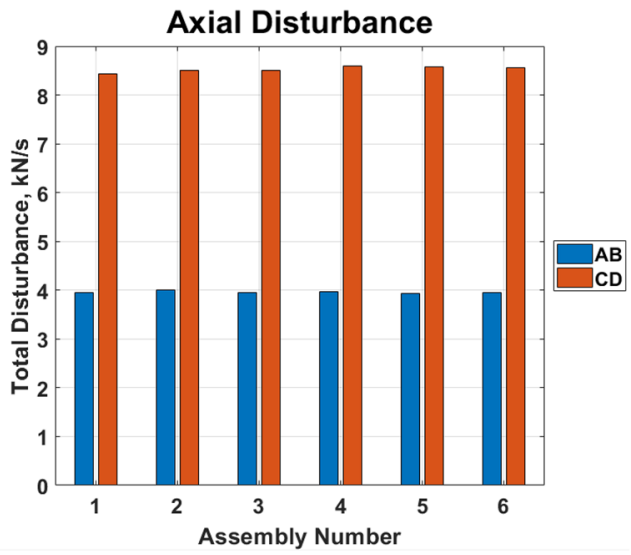

(b) 

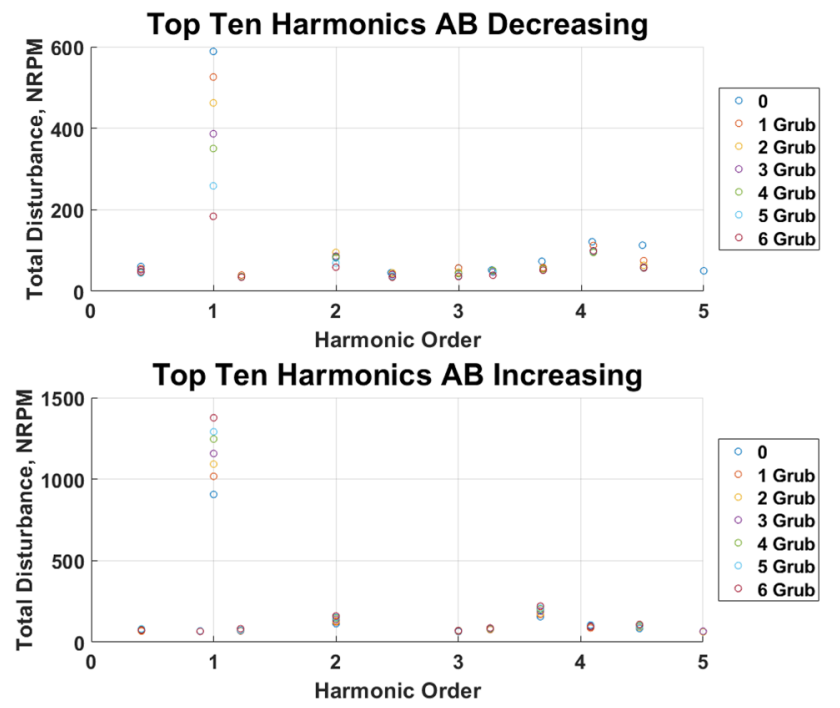

Fig. 27 Top ten harmonics found in the unbalance tests using bearings $\mathrm{AB}$

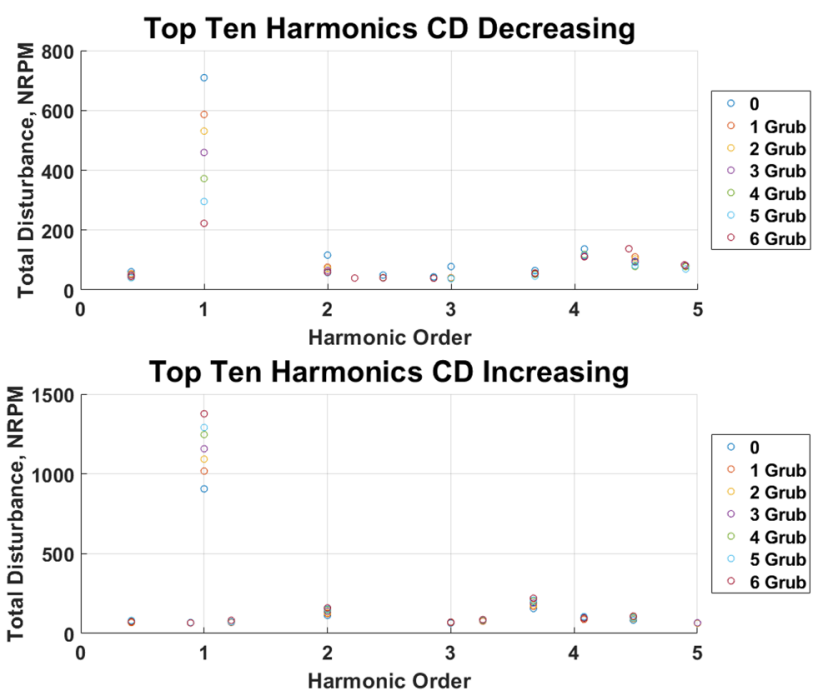

Fig. 28 Top ten harmonics found in the unbalance tests using bearings $\mathrm{CD}$
3. As each assembly used the same process and equipment, the frequency of each disturbance remained mostly constant as expected, however, a significant variation between the amplitudes of the bearing disturbances was observed. This was seen both at a top and harmonic level in all three bearing sets. Without further investigations, any potential explanation at this stage can be considered speculative. However, as the bearings used in these tests were made to a high standard as required for aerospace applications, there are several potential explanations for this phenomenon. For example, due to tolerances in the tools used there may be slight variations in the preload which could have had an effect on the contact angle of the running track. Because of these small changes in preload conditions, changes in features like the structural modes might not be easily noticeable. However, due to the size of any imperfections this shift could make the difference between the ball running on the defect or clipping the edge having a major impact on the surface area of the interaction. As shown in [13], the general shape or edge can influence the disturbance amplitude. Also, this is can be considered due to the slight order shift seen by the FBOR disturbances shown in Fig. 21. Some other additional explanations could be variation in sliding friction, angular tolerance and rotational position of the bearing and lateral tolerance of the preload spring which could affect the loading centre.

From the results and conclusions in his study, it is clear that additional work is needed to create an explanation behind the cause of the variation in bearing disturbance amplitudes. Some speculative explanations have been discussed with many revolving around the defect geometry and running track, both parameters could be measured through microscopy. From literature some models have been created to approximate the contact region between a rolling element and a raceway, by comparing that to the measured running track would provide insight into some variation between assemblies. Taking a more analytical approach, simulations can be done to model different
Table 10 Mean and standard deviation of harmonics $3.80,4.06$ and 4.51

\begin{tabular}{|c|c|c|c|c|c|}
\hline \multirow[t]{2}{*}{ Scenario } & \multirow[t]{2}{*}{ Harmonic } & \multicolumn{3}{|l|}{$\mathrm{AB}$} & \multirow{2}{*}{$\begin{array}{l}\text { CD } \\
\text { Standard deviation }\end{array}$} \\
\hline & & Mean (NRPM) & $\begin{array}{l}\text { Standard } \\
\text { deviation }\end{array}$ & Mean (NRPM) & \\
\hline \multirow[t]{3}{*}{ Decreasing } & 3.8 & 6.056 & 0.762 & 4.857 & 0.1917 \\
\hline & 4.06 & 12.525 & 1.502 & 15.915 & 2.341 \\
\hline & 4.5 & 6.917 & 0.755 & 8.418 & 2.470 \\
\hline \multirow[t]{3}{*}{ Increasing } & 3.8 & 3.349 & 0.158 & 12.262 & 2.860 \\
\hline & 4.06 & 16.391 & 1.439 & 9.600 & 0.541 \\
\hline & 4.5 & 2.834 & 0.077 & 9.859 & 0.217 \\
\hline
\end{tabular}


Fig. 29 FBOR comparison

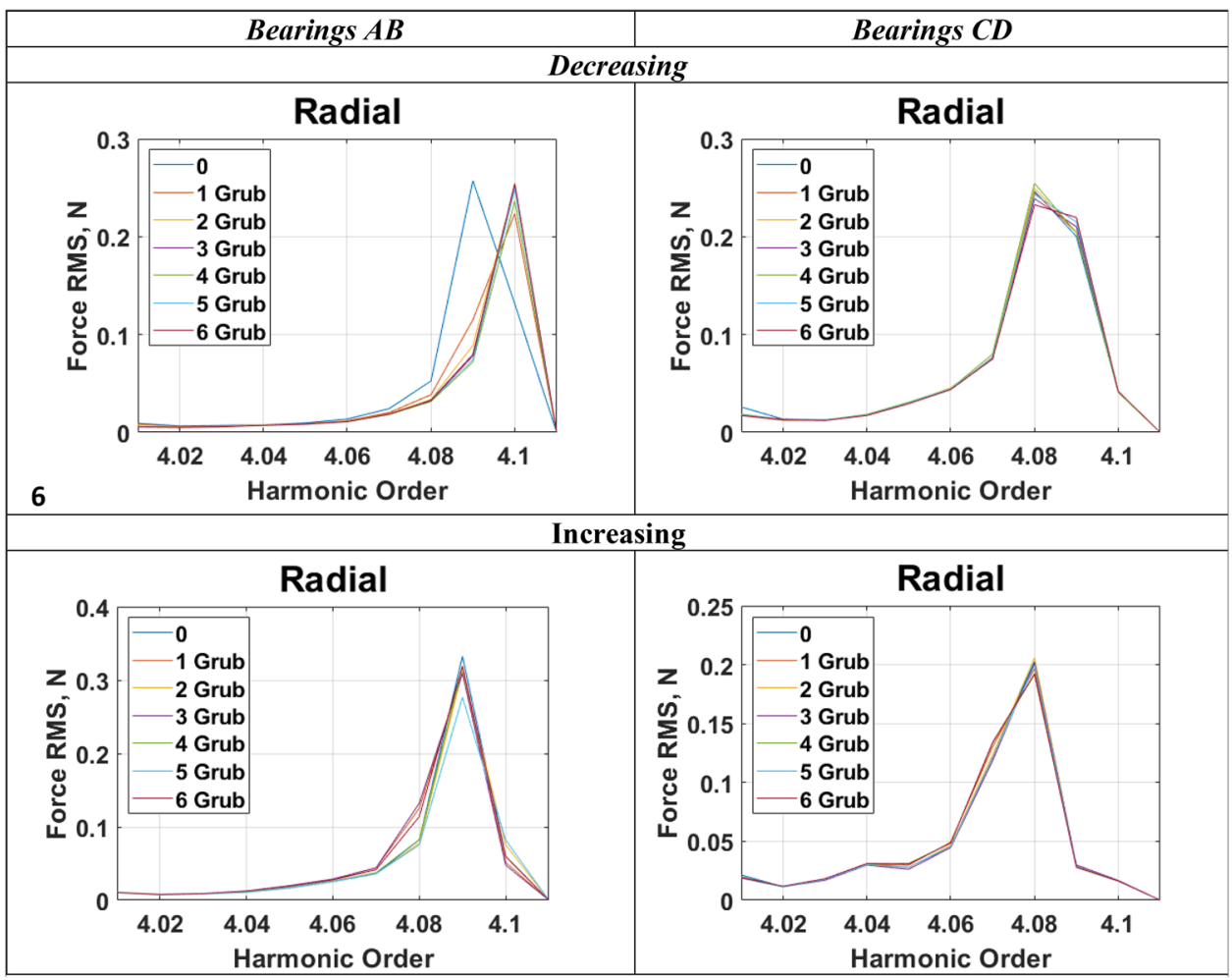

defects and how changing the geometry can affect the resulting disturbances. However, to investigate the other speculative causes, modifications to the test rig or assembly process may be required to reduce tolerance build up.

Funding Funding was supplied by the university of surrey, Surrey Satellite Technologies Ltd and the European Space Agency.

Availability of data and material Partial availability (Most of the data was generated under an ESA funded Study).

Code availability Not applicable.

\section{Compliance with ethical standards}

\section{Conflict of interest None.}

Open Access This article is licensed under a Creative Commons Attribution 4.0 International License, which permits use, sharing, adaptation, distribution and reproduction in any medium or format, as long as you give appropriate credit to the original author(s) and the source, provide a link to the Creative Commons licence, and indicate if changes were made. The images or other third party material in this article are included in the article's Creative Commons licence, unless indicated otherwise in a credit line to the material. If material is not included in the article's Creative Commons licence and your intended use is not permitted by statutory regulation or exceeds the permitted use, you will need to obtain permission directly from the copyright holder. To view a copy of this licence, visit http://creativecommons.org/licenses/by/4.0/.

\section{References}

1. Zhang, Z., Aglietti, G.S., Ren, W.: Coupled microvibration analysis of a reaction wheel assembly including gyroscopic effects in its accelerance. J Sound Vib 332(22), 5748-5765 (2013)

2. Addari, D., Aglietti, G.S., Remedia, M.: Dynamic mass of a reaction wheel including gyroscopic effects: an experimental approach. AIAA J 55(1), 274-285 (2017)

3. Xiongfei, L., Wei, C., Li, X., Cheng, W.: Research on microvibrations generated by control moment gyroscope on flexible interface based on dynamic substructure method. Int J Aerosp Eng 2018, 1-17 (2018)

4. De Lellis, S., Stabile, A., Aglietti, G.S., Richardson, G.: A semiempirical methodology to characterise a family of microvibration sources. J Sound Vib 448, 1-18 (2019)

5. Chapel, J., et al.: Guidance, navigation, and control performance for the GOES-R spacecraft. CEAS Sp J 7(2), 87-104 (2015)

6. ECSS.: ECSS-E-HH-32-26A, Spacecraft mechanical loads analysis handbook, 1st ed European Cooperation for Space Standardization, Noordwijk (2013).

7. Hinque, V., Seiler, R.: Dynamic behavior of ball bearings under axial vibration. Aerosp Mech Symp 2, 83-96 (2018)

8. Karimian, A., Shokrollahi, S., Yousefi, S., Aghalari, A.: Analytical disturbance modeling of a flywheel due to statically and dynamically unbalances. J Math Comput Sci 09(02), 139-148 (2014)

9. Zhang, Z., Aglietti, G.S., Ren, W., Addari, D.: Microvibration analysis of a cantilever configured reaction wheel assembly. Adv Aircr Spacecr Sci 1(4), 379-398 (2014)

10. Cheon, D.I., Jang, E.J., Oh, H.S.: Reaction wheel disturbance reduction method using disturbance measurement table. J Astron Sp Sci 28(4), 311-317 (2011) 
11. Elias, L.M., Dekens, F.G., Basdogan, I., Sievers, L.A., Neville, T.: Methodology for modeling the mechanical interaction between a reaction wheel and a flexible structure. Interferom Sp 4852, 541 (2003)

12. Masterson, R., Miller, D., Groga, R.: Development of empirical and analytical reaction wheel disturbance models. Massachusetts Institute of Technology, Massachusetts (1999)

13. Liu, J., Shao, Y., Zuo, M.J.: The effects of the shape of localized defect in ball bearings on the vibration waveform. Proc Inst Mech Eng Part KJ Multi-body Dyn 227(3), 261-274 (2013)

14. Kong, F., Huang, W., Jiang, Y., Wang, W., Zhao, X.: A vibration model of ball bearings with a localized defect based on the hertzian contact stress distribution. Shock Vib 2018, 1-14 (2018)

15. Grajales, J.A., Lopez, J.F., Quintero, H.F.: Ball bearing vibrations model: development and experimental validation. Ing. y Compet. 16(2), 279-288 (2014)

16. Yang, R., Jin, Y.L., Hou, L., Chen, Y.S.: Super-harmonic resonance characteristic of a rigid-rotor ball bearing system caused by a single local defect in outer raceway. Sci. China Technol. Sci. 61(8), 1184-1196 (2018)

17. Zhou, W., Li, D., Luo, Q., Liu, K.: Analysis and testing of microvibrations produced by momentum wheel assemblies. Chinese J. Aeronaut. 25(4), 640-649 (2012)

18. Addari, D.: A Semi-empirical approach for the modelling and analysis of microvibration sources on-board spacecraft. University of Surrey, Guildford (2016)

19. Zhao, Y., Sun, J., Tian, H.: Development of methods identifying parameters in reaction wheel assembly disturbance model. Aircr. Eng. Aerosp. Technol. 78(4), 326-330 (2006)

20. Kim, D.K.: Micro-vibration model and parameter estimation method of a reaction wheel assembly. J. Sound Vib. 333(18), 4214-4231 (2014)

21. MacCamhaoil, M.: Static and dynamic balancing of rigid rotors, p. 2. Bruel and Kjaer, Naerum (1989)

22. Kuiper, H., Dolkens, D.: A cutting edge 6U CubeSat ADCS design for earth observation with sub-meter spatial resolution at 230-380 km altitude. CEAS Sp J 12(4), 613-621 (2020)

23. Wang, H., Han, Q.K., Luo, R.Z., Qing, T.: Dynamic modeling of moment wheel assemblies with nonlinear rolling bearing supports. J. Sound Vib. 406, 124-145 (2017)
24. De Lellis, S.: A methodology to account for dynamic variability in micro-vibration analysis of satellites. University of Surrey, Guildford (2019)

25. Rahnejat, H., Gohar, R.: The vibrations of radial ball bearings. Proc Inst Mech Eng Part C Mech Eng Sci 199(3), 181-193 (1985)

26. Harsha, S.P.: Nonlinear dynamic analysis of rolling element bearings due to cage run-out and number of balls. J. Sound Vib. 289(1-2), 360-381 (2006)

27. Jacobs, W., Boonen, R., Sas, P., and Moens, D.: "Measuring the rigid body behaviour of a deep groove ball bearing setup," in International Conference on Noise and Vibration Engineering 2012, ISMA 2012, including USD 2012: International Conference on Uncertainty in Structure Dynamics, 1, pp. 715-725 (2012)

28. Tandon, N., Choudhury, A.: Review of vibration and acoustic measurement methods for the detection of defects in rolling element bearings. Tribol. Int. 32(8), 469-480 (1999)

29. Lacey, S.J.: an overview of bearing vibration analysis. Maint. Asset Manag. 23(6), 32-42 (2008)

30. Kulkarni, P. G., and Sahasrabudhe, A. D.: "A theoretical model for studying vibrations of ball bearing with localised defects on inner race using cubic hermite spline," in 2015 International Conference on Industrial Instrumentation and Control (ICIC), Icic, pp. 121-126 (2015)

31. Sung, K., Shirley, P., Baer, S.: Essential Concepts Essentials of Interactive Computer Graphics, pp. 154-156. CRC Press, Boca Raton (2008)

32. Rai, A., Upadhyay, S.H.: A review on signal processing techniques utilized in the fault diagnosis of rolling element bearings. Tribol. Int. 96, 289-306 (2016)

33. Patil, M.S., Mathew, J., Rajendrakumar, P.K., Desai, S.: A theoretical model to predict the effect of localized defect on vibrations associated with ball bearing. Int. J. Mech. Sci. 52(9), 1193-1201 (2010)

Publisher's Note Springer Nature remains neutral with regard to jurisdictional claims in published maps and institutional affiliations. 